Pamph

Philos

Logic

s

\title{
OUNDATIONS OF FORMAL LOGIC
}

BY

HENRY BRADFORD SMITH
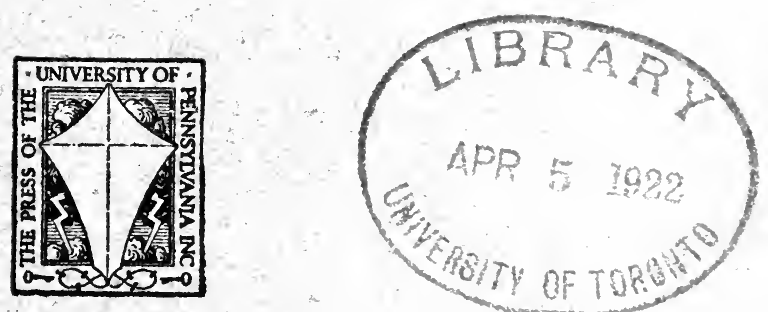

PHILADELPHIA

PRESS OF THE UNIVERSITY OF PENNSYLVANIA 





\section{FOUNDATIONS OF FORMAL LOGIC}

BY

HENRY BRADFORD SMITH

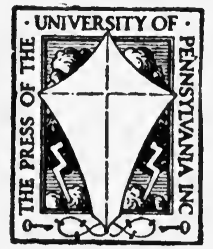

PHILADELPHIA

PRESS OF THE UNIVERSITY OF PENNSYLVANIA 



\section{PREFACE}

The edition of his Primer of Logic being exhausted, the writer is forced to prepare another outline for classroom use. Only parts of that text, however, have been retained and these have received a methodical rearrangement and expansion. Two chapters of the work, Non-Aristotelian Logic, appear again but modified in detail. To these have been added historical notes and citations and three new final chapters and the text has been illustrated by a number of diagrams.

The writer has again to express his indebtedness to Professor Singer for his introduction to the method which is here employed. This indebtedness is to be referred not only to the Syllabus of his lectures (reprinted pp. 46-52 in the writer's Letters on Logic) but also to many hints thrown out in private discussion.

The present work, as its title suggests, does not pretend that its system is completely developed. Its chief concern is with the foundations, upon which a theory may be built. Its solutions would have been carried farther, if the writer could have found his way through to the end.

H. B. S. 



\section{TABLE OF CONTENTS}

\section{CHAPTER I}

PAGES

§ 1-6 Forms of proposition recognized by the logician. . 7-13

CHAPTER II

8-11 Relations of "better" and "worse." Immediate inference.......................... 14-18

CHAPTER III

\$12-16 Moods of the syllogism.

CHAPTER IV

\$17-22 General solution of the sorites $26-32$

CHAPTER V

\$23-31 Foundations of the calculus. $33-40$

CHAPTER VI

\$32-35 General solution of the syllogism $41-44$

CHAPTER VII

\$36-40 On certain supposed fallacies. $45-49$

CHAPTER VIII

\$ 41-42 Alternative systems. Non-Aristotelian logic... 50-51 CHAPTER IX

\$4-46 The classical system and its verification ...... 52-56 



\section{CHAPTER I}

\$1. The problem of a deductive science is to define its elements, the objects of which it treats, by an enumeration of their formal properties. These properties are to be found within the system of which these objects are the parts. The task of logic ${ }^{1}$ is, then, to develop its own system by constructing all the true and all the untrue propositions into which its elements enter exclusively.

According to Schröder, if $a, b, c$.. be a set of indefinables and $R$ a relation, then $a, b, c \ldots R$ are said to form a system, provided $p R q$ stand for an assertion that must be either true or false and can not be both true and false, $-p$ and $q$ representing any members of the set, $a$, b, c... (See Schröder's Abriss der Algebra der Logik, by Dr. Eugen Müller, Leipzig, 1909, p. 18.)

\$2. We shall begin with an enumeration of the forms with which logic deals.

The Categorical forms are composed of terms and relationships and are represented by the following propositional functions:

$$
\begin{aligned}
& a(a b)=\text { All } a \text { is all } b, \\
& \beta(a b)=\text { Some } a \text { is some } b, \\
& \gamma(a b)=\text { All } a \text { is some } b, \\
& \epsilon(a b)=\text { No } a \text { is } b .
\end{aligned}
$$

These are called propositional functions because each one is true only for some, not for all meanings of $a$ and $b$; because their truth or untruth depends upon the meaning of $a$ and $b$. Whenever it is desired to designate indifferently any one of these (i. e., to leave it unsettled which one is meant) the notation $x(a b)$, $y(a b)$, etc., will be employed. In the proposition $x(a b)$ the terms are the subject $a$ and the predicate $b$ and the term order is the order subject-predicate. Whenever the term order is unsettled a comma will appear between the terms. Thus $x(a, b)$ may mean either $x(a b)$ or $x(b a)$. The relationships in the categorical forms are the copula is and the adjectives of quantity, all, some and no.

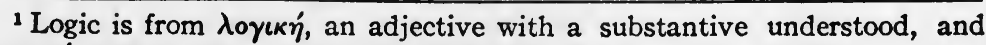

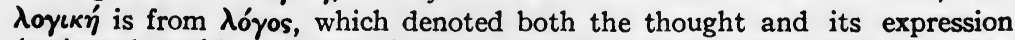
(ratio and oratio). This ambiguity passed into the meaning of the derivative $\lambda \circ \gamma \bullet \dot{y}^{\prime}$ and led indirectly to a dispute as to whether Logic deals with the laws of thought or with the laws of the expression of thought. 
The word some is ambiguous in ordinary speech, meaning either some at least, possibly all or some at most, some at least, not all. It is this latter interpretation, which we shall assume to be forced upon us by the propositions which we say shall be true or untrue in our science.

In $1846 \mathrm{Sir}$ William Hamilton published the prospectus of an essay on a New Analytic of Logical Forms, which revived the question as to whether or not the quantity of the predicate should be made explicit. The chief difficulties of his system result from the ambiguity of the meaning of some, from the effort to make every form of categorical expression simply convertible and from the seemingly curious effort to establish an order of better and worse among the relations connecting subject and predicate. Four of Hamilton's eight forms are redundant. Those that are essential are represented here by the letters, $a, \beta, \gamma$ and $\epsilon$.

$\S 3$. The terms, $a$ and $b$, stand for classes, for a group of objects conceived by the aid of a common property. Every substantive in the language is the symbol for such a group. There is a certain analogy between the manner in which closed areas overlap and the manner in which classes overlap, which was first pointed out by the mathematician Euler. This analogy often breaks down, as might be expected, for some limiting case and has perhaps led the logician astray as often as it has aided him. It will prove to be invaluable, however, in enabling us to attach a preliminary meaning to our symbols of relationship.

"As a general notion contains an infinite number of individual objects, we may consider it a space in which they are all contained. Thus for the notion of man we form a space, in which we conceive all men to be comprehended. For the notion of mortal we form another in which we conceive everything mortal to be comprehended. And when I affirm all men are mortal it is the same thing with affirming that the first figure is contained in the second. . . . These circles, or rather these spaces, for it is of no importance of what figure they are, are extremely commodious for facilitating our reflections on this subject, and for unfolding all the boasted mysteries of logic, which that art finds it so difficult to explain; whereas by means of these signs the whole is rendered sensible to the eye." (Letters of Euler addressed to a German Princess, by David Brewster, New York, 1846, Vol. I, pp. 339-341.)

The diagrammatic representation of the categorical forms is given below. 
All $a$ is all $b$

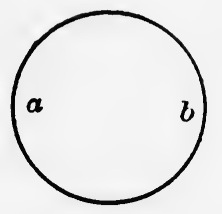

Some $a$ is some $b$

All $a$ is some $b$

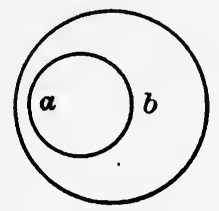

All $b$ is some $a$

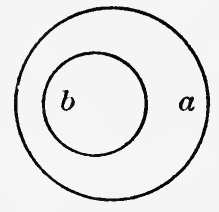

No $a$ is $b$

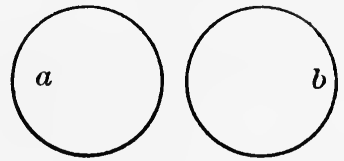

Assuming now that these forms exhaust all of the modes in which two closed areas may overlap, it will seem natural to declare, on the ground of our analogy, that any two classes, $a$ and $b$, must be related in one, and can not be related in more than one, of these five ways. The assertion that $a$ and $b$ must realize one of these possibilities we shall term the propositional universe. The assertion that $a$ and $b$ must realize more than one of these possibilities we shall term the propositional null. The former assertion will then appear to be true for all meanings of the terms and the latter assertion will appear to be untrue for all meanings of the terms.

$\S 4$. The remaining forms of proposition, which are recognized by the logician, are: 
The Hypothetical form,

if $x$ (is true) then $y$ (is true),

$=x$ implies $y=x<y$,

$x$ does not imply $y=(x<y)^{\prime}$;

The Conjunctive form,

$x$ (is true) and $y$ (is true),

$=x y$;

The Disjunctive form,

either $x$ (is true) or $y$ (is true),

$=x+y$.

Here $x$ and $y$ in turn stand for any sort of proposition. If they happen to be categorical forms, then we should replace the abbreviations above by the more definite notation, $x(a, b)<y(a, b)$, $x(a, b) y(a, b), x(a, b)+y(a, b)$. The untruth of $x$ will be denoted by $x^{\prime}$, the untruth of $x^{\prime}$ by $x^{\prime \prime}$.

The relations of inclusion and implication are usually rendered by Peano's sign of a flat " $C$ " opening to the left, the initial letter of the word contains; or by Schröder's half bracket opening to the right and drawn through an equality sign. The symbol of the text is a simplification of the one employed by Schröder, only the upper half being retained.

\$5. The word "true" never connotes possibly true or true in some instances. It means necessarily true, true for all cases, true for all meanings of the terms. The following propositions may be verified at once "empirically" by the aid of Euler's diagrams. If the student will examine each illustration with care, he will begin to attach a meaning to the symbolism. Let him observe that the figure in each case represents the part to the left of the implication sign as true and the part to the right of the implication sign as true at the same time.

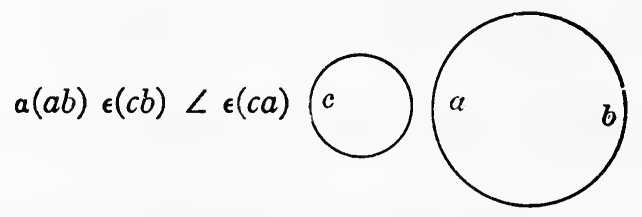




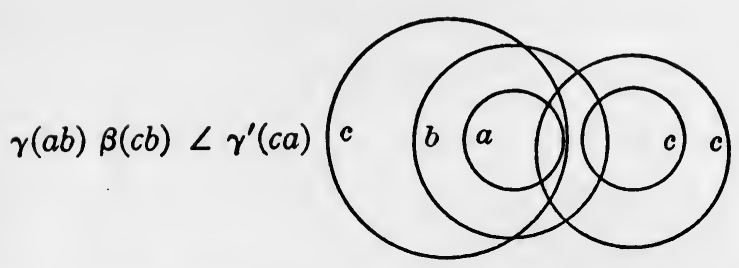

"Inference does not give us more than there was before: but it makes us see more than we saw before. . . . The homely truth that no more can come out than was in, . . . has been applied to logic, and even to mathematics, in depreciation of their rank as branches of knowledge. . . . and hence some have spoken as if in studying how to draw the conclusion, we are studying to know what we knew before. . . .

"The study of logic, . . . considered relatively to human knowledge, stands in as low a place as that of the humble rules of arithmetic, with reference to the vast extent of mathematics and their physical applications. Neither is the less important for its lowliness: but it is not every one who can see that." (De Morgan, Formal Logic, London, 1847, pp. 44-46.)

The word "untrue" means not necessarily true, not true in all instances. Accordingly, in order to establish the untruth of a given proposition, it will be enough to point to a single instance of its being untrue. The diagrams which follow will establish "empirically" the untruth of the proposition to which each one corresponds. The fact to be noticed here is that the figure in each case represents the part to the left of the implication sign as true and the part to the right of the implication sign as false at the same time.

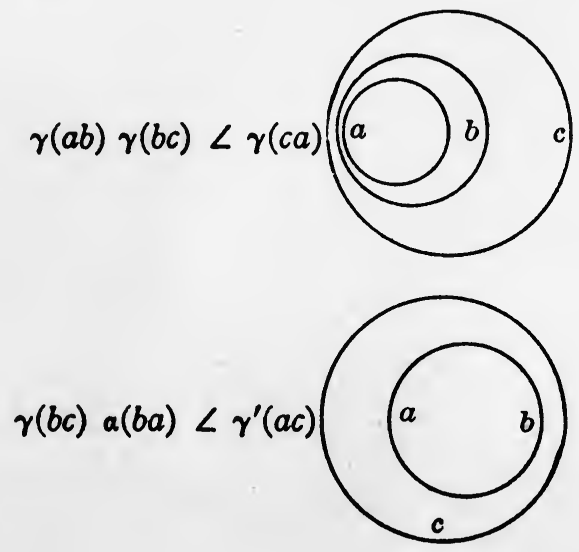


$\S 6$. If we refer again to the five possible modes of representing the formal relationship of $a$ to $b$, viz.,
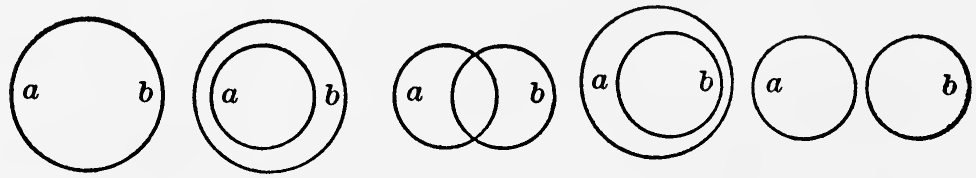

it will be easy to understand what is contained in the statement, that $x(a b)$ is false. This will be the same as to assert that the possibility for which $x(a b)$ stands, is to be left out of the universe and the sum of the remaining possibilities asserted to be true. The prime over $x(a b)$ may be conceived as an operator which strikes out the corresponding diagram. Thus $\epsilon^{\prime}(a b)$ will be represented precisely by the disjunction:

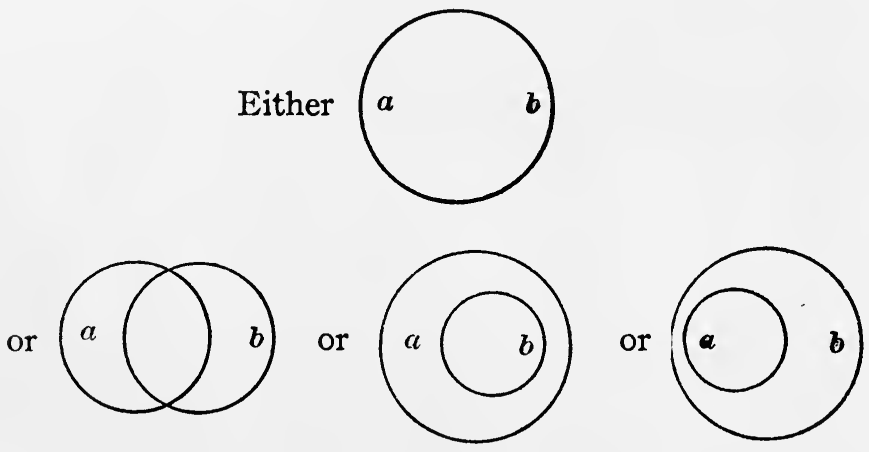

Whenever the conjunction of two or more propositions stands for an impossibility, their product is said to be null. Thus, the product of $a$ and $\beta$ is null, but the product of $a^{\prime}$ and $\beta^{\prime}$ is not null.

Whenever the disjunction of two or more propositions exhausts all of the possibilities that there are, their sum is said to be universe. Thus, the sum of $a^{\prime}$ and $\beta^{\prime}$ is universe, but the sum of $a$ and $\beta$ is not universe.

The following definitions are of importance for our subsequent theory:

(1) If the logical sum of two categorical forms is the universe and their logical product is null, the two are said to be contradictory forms.

(2) If the logical sum of two categorical forms does not exhaust the universe but their logical product is null, the two are said to be contrary forms. 
(3) If the logical sum of two categorical forms is the universe but their logical product is not null, the two are said to be subcontrary forms.

(4) If the logical sum of two categorical forms does not exhaust the universe and their logical product is not null, the two are said to be subalternate forms.

Suppose, now, that $\mathrm{k}(a b)$ and $\mathrm{w}(a b)$ designate any one of the unprimed categorical forms but cannot designate the same form, $\gamma(a b)$ and $\gamma(b a)$ being considered distinct, then the following statements may be verified at once as the result of a complete induction of all of the possibilities:

(1) $\mathbf{k}(a b)$ and $\mathbf{k}^{\prime}(a b)$ are contradictory;

(2) $\mathbf{k}(a b)$ and $\mathbf{w}(a b)$ are contrary;

(3) $\mathbf{k}^{\prime}(a b)$ and $\mathbf{w}^{\prime}(a b)$ are subcontrary;

(4) $\mathbf{k}(a b)$ and $\mathrm{w}^{\prime}(a b)$ are subaltern. 


\section{CHAPTER II}

$\S 7$. At this point in our theory we shall introduce certain relations, which will be termed the distinctions of better and worse, following a suggestion of Sir William Hamilton's making (Lectures on Logic, Appendix, p. 536). It will turn out in the sequel that these assumed indefinables may be dispensed with. A study of them, however, has an historical interest and will serve the purpose of making meaningful the process of deduction. They fulfil the spirit rather than the letter of Hamilton's thought.

Let us begin by inventing symbols to denote our distinctions, i. e.,

$$
\begin{aligned}
& x / y=x \text { is singly worse than } y, \\
& x / / y=x \text { is doubly worse than } y, \\
& x / / y=x \text { is trebly worse than } y
\end{aligned}
$$

and let us add the following:

Definition.-In the propositions, $x / y, x / / y$ and $x / / / y$, the relation connecting $x$ and $y$ is known as the worse-relation.

Definition.-Singly worse, doubly worse and trebly worse are known as the three degrees of the worse-relation.

Let us suppose now that $x$ and $y$ can represent only the categorical forms and let us imagine $x$ and $y$ to take on in all possible ways the values, $a, \beta, \gamma$ and $\epsilon$. There will then be sixteen possible propositions of each type, $x / y, x / / y$ and $x / / ! y$, obtained by permuting the letters two at a time and by taking each letter once with itself. If the symbol of relationship be omitted and each proposition be exhibited as a simple combination of the two letters, then each set of sixteen would appear thus:

$\begin{array}{llll}\alpha a & \beta a & \gamma \alpha & \epsilon a \\ a \beta & \beta \beta & \gamma \beta & \epsilon \beta \\ \alpha \gamma & \beta \gamma & \gamma \gamma & \epsilon \gamma \\ \alpha \epsilon & B \epsilon & \gamma \epsilon & \epsilon \epsilon\end{array}$

All of the propositions of this set taken together are known as the array of whichever type, $x / y, x / / y$ or $x / / / y$, they particularize. Each proposition of the array is known as a mood of the array. True moods of the array are known as valid moods of the array. The remaining moods are known as invalid moods of the array. 
\$ 8. The three arrays, once the valid and invalid moods have been established, constitute a species of definition of the three degrees of the worse-relation, for they exhaust all of the propositions into which this relationship may enter. We proceed to formulate certain rules and to assume certain postulates by the aid of which all of the moods, valid and invalid, may be deduced.

Definition 1.-In the propositions, $x / y, x / / y$ and $x / / / y, x$ is called the inferior and $y$ is called the superior form.

Definition 2.-Trebly worse is singly worse than doubly worse and doubly worse is singly worse than singly worse.

Rule 1.-If in any valid mood inferior form and worse-relation be each made singly worse, a valid mood will result.

Postulate $1 .-\beta / a$ is a valid mood.

Postulate $2 .-\gamma / \beta$ is a valid mood.

Postulate 3. $-\epsilon / \gamma$ is a valid mood.

Applying rule 1 to postulate 1 , we obtain at once

Theorem $1 .-\gamma / / a$ is a valid mood.

(Postulate 2 and Definitions 1 and 2).

Applying rule 1 to theorem 1, we obtain at once

Theorem 2. $-\epsilon / / / a$ is a valid mood.

(Postulate 3 and Definitions 1 and 2.)

Applying rule 1 to postulate 2, we obtain at once

Theorem 3. $\epsilon / / \beta$ is a valid mood.

(Postulate 3 and Definitions 1 and 2.)

The rules and postulates for the deduction of the invalid moods are given below. The derivation of the thirty-nine theorems is left as an exercise for the student.

Definition 3.-If $x$ is worse than $y$, then $y$ is said to be better than $x$ in the corresponding degree.

Rule 2.-If in any invalid mood superior and inferior form be each made singly better or each made singly worse, an invalid mood will result.

Rule 3.-If any invalid mood inferior form and worse-relation be each made singly worse, an invalid mood will result.

Rule 4.- If in any invalid mood superior form be made trebly worse, an invalid mood will result.

Postulate 4.- $a / \gamma$ is an invalid mood.

Postulate 5. $-\gamma / a$ is an invalid mood.

Postulate 6. $-\epsilon / a$ is an invalid mood.

Theorems.-The remaining (39) invalid moods. 
The "order" of better and worse among the four forms being now unambiguously established by postulate and theorem, we may exhibit our result schematically thus:

\section{Best $\quad \alpha-\beta-\gamma-\epsilon \quad$ Worst}

\$9. In the hypothetical forms, $x<y$ and $(x<y)^{\prime}$, the part $x$ to the left of the implication sign, is called the antecedent and the part $y$ to the right of the implication sign is called the consequent.

Here $x$ and $y$ may stand for any sort of proposition but if each one happens to represent a single categorical form, we should then replace $x<y$ and $(x<y)^{\prime}$ by the more definite notation, $x(a, b)$ $\angle y(a, b)$ and $\{x(a, b) \angle y(a, b)\}^{\prime}$. Any implication of this specific type is known as immediate inference.

A difference between two forms of inference which is dependent on term-order alone, is known as a difference of figure. Thus, $x(a, b)<y(a, b)$ may have either one of two forms. If the termorder in the antecedent is the same as the term order in the consequent, i. e., if $x(a, b)<y(a, b)$ be written

$$
\begin{aligned}
& \text { either } x(a b)<y(a b), \\
& \text { or } \quad x(b a)<y(b a),
\end{aligned}
$$

then $x(a, b) \angle y(a, b)$ is said to be expressed in the first figure of immediate inference. If the term-order in the antecedent is the reverse of the term-order in the consequent, i. e., if $x(a, b) \angle y(a, b)$ be written

$$
\begin{aligned}
\text { either } x(a b) & <y(b a), \\
\text { or } \quad x(b a) & <y(a b),
\end{aligned}
$$

then $x(a, b) \angle y(a, b)$ is said to be expressed in the second figure of immediate inference.

Just as the comma between the terms means that the termorder is not settled, so the $x$ in $x(a, b)$ and the $y$ in $y(a, b)$ mean that the categorical form for which $x(a, b)$ or $y(a, b)$ stands is undetermined. Suppose now that we should particularize $x$ and $y$, that is, allow them to take on in every possible way their four specific values, $a, \beta, \gamma$ and $\epsilon$. There will evidently result sixteen distinct moods of the array $x(a, b) \angle y(a, b)$ in each one of the two figures.

$\S 10$. If $x(a b) \angle x(b a)$ is a valid mood of immediate inference, then $x(a b)$ is said to be a convertible form. The operation of simple 
conversion consists in the interchange of subject and predicate. Referring again to the diagrams of the last chapter, it will seem natural to attach this property of simple convertibility to $a, \beta$ and $\epsilon$. Employing this terminology we should then say that $a, \beta$ and $\epsilon$ are convertible forms or that $a, \beta$ and $\epsilon$ are simply convertible. In order to give expression to this fact, let the following assumptions be granted:

Postulate 1. $-a(a b) \angle a(b a)$ is a valid mood.

Postulate 2. $-\beta(a b)<\beta(b a)$ is a valid mood.

Postulate 3. $-\gamma(a b)<\gamma(b a)$ is an invalid mood.

Postulate 4. $-\epsilon(a b)<\epsilon(b a)$ is a valid mood.

We shall now set down the rules for the deduction of the valid moods of immediate inference:

Rule 1.-If in any valid mood the subject and predicate be interchanged in a form that is simply convertible, a valid mood will result.

Rule 2.-If in any valid mood of the first figure the antecedent and the consequent be each made singly worse, a valid mood will result.

Theorem 1. $-a(a b) \angle a(a b)$ is a valid mood.

(Postulate 1 and Rule 1.)

Theorem 2. $-\beta(a b) \angle \beta(a b)$ is a valid mood,

(Theorem 1 and Rule 2.)

Theorem 3. $-\gamma(a b) \angle \gamma(a b)$ is a valid mood, (Theorem 2 and Rule 2.)

Theorem 4.- $\epsilon(a b) \angle \epsilon(a b)$ is a valid mood, (Theorem 3 and Rule 2.)

The rules and postulates for the deduction of the invalid moods are given below. The derivation of the twenty-one theorems is left as an exercise for the student.

Rule 1.-If in any invalid mood the subject and predicate be interchanged in a form that is simply convertible, an invalid mood will result.

Rule 2.-If in any invalid mood of the first figure, the antecedent and the consequent be each made singly worse, an invalid mood will result.

Rule 3.-If in any invalid mood of the first figure the antecedent and the consequent be interchanged, an invalid mood will result. 
Postulate 5. $-a(a b) \angle \beta(a b)$ is an invalid mood.

Postulate 6. $-a(a b)<\gamma(a b)$ is an invalid mood.

Postulate 7. $-a(a b)<\epsilon(a b)$ is an invalid mood.

Theorems. - The other (21) invalid moods.

$\S 11$. We may also formulate rules for the immediate detection of the invalid moods, as follows:

Rule 1.-If the antecedent be worse than the consequent the mood is invalid.

Rule 2.-If the antecedent be better than the consequent the mood is invalid.

Definition.-Distributed terms are those modified by the adjectives all or no, i. e., the subject of $a, \gamma$ and $\epsilon$ and the predicate of $a$ and $\epsilon$. That the predicate of $\epsilon$ is distributed may be seen at once from the property of simple convertibility of this form.

Rule 3.-If a term which is distributed in the consequent, be undisturbed in the antecedent, the mood is invalid.

These rules are both necessary and sufficient for the purpose which they effect, as will appear from the following consideration: They are sufficient, because they declare all the moods not already found to be valid, to be invalid. Each one is necessary, because we can point to at least one invalid mood, which falls uniquely under each rule. 


\section{CHAPTER III}

$\S 12$. The array which will next engage our attention is of a somewhat more general character than the one of immediate inference, and we may begin not with a definition of it in abstract terms but with an examination of specific examples.

Let us consider the proposition, $a(b a) \beta(c b) \angle \beta(c a)$, A single one of Euler's diagrams will be enough to furnish a representation of the two factors conjoined in the antecedent.

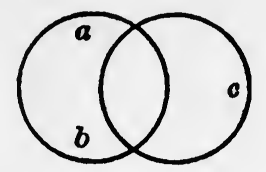

It will be noticed that this figure verifies not only the antecedent but the consequent as well, so that the implication would appear to be a valid one. It may be observed in general, that whenever every mode of representing the terms as related in the antecedent is also a mode of representing the terms as related in the consequent, then the implication is, at least empirically true.

In order to have before us an instance in which this double verification breaks down, let us examine the proposition, $\epsilon(a b) \gamma(b c)$ $\angle \epsilon(c a)$. The diagram of the antecedent as a whole will appear as in the figure below.

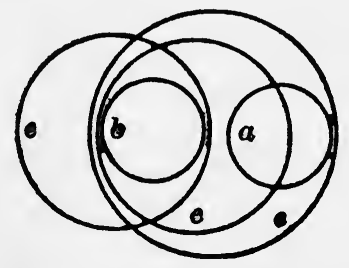

But here there exist two cases in which the consequent is not verified. Accordingly, if $a, b$ and $c$ are related as in the antecedent, it does not follow that $a$ and $c$ are related as in the consequent and the empirical untruth of the implication is manifest.

\$13. The type of proposition which our two examples particularize, is known as the syllogism and its igeneral expression is 
$x(a, b) y(b, c)<z(c, a)$. Let us begin our study of this form of inference by determining the various possible ways of arranging the terms. These varieties of figure will manifestly be not more than eight in number, viz.,

$\begin{array}{llll}\mathrm{ba} & \mathrm{ab} & \mathrm{ba} & \mathrm{ab} \\ \mathrm{cb} & \mathrm{cb} & \mathrm{bc} & \mathrm{bc} \\ -\mathrm{ca} & - & - & - \\ \mathrm{ca} & \mathrm{ca} & \mathrm{ca} \\ \mathrm{cb} & \mathrm{ab} & \mathrm{ba} & \mathrm{ab} \\ \overline{\mathrm{ac}} & - & \mathrm{bc} & \mathrm{bc} \\ \mathrm{ac} & - & -\end{array}$

The two forms, $x(a, b)$ and $y(b, c)$, conjoined in the antecedent may evidently be written in the order $x y$ or in the order $y x$, for the conjunctive relation of logic is, as we say, commutative. Let it now be agreed as a matter of convention always to write first the form which contains the predicate of the consequent. To accord with this convention the second set of four term-orders above will have to be rearranged thus:

$\begin{array}{cccc}c b & c b & b c & b c \\ \text { ba } & a b & b a & a b \\ - & - & - & - \\ \text { ac } & \text { ac } & \text { ac } & \text { ac }\end{array}$

Let us now draw a horizontal line which will join the two terms in the first factor of the antecedent and a line which will join the term $b$ above with the term $b$ below. The "figures" so constructed, when isolated from the letters which they join, would then appear thus:
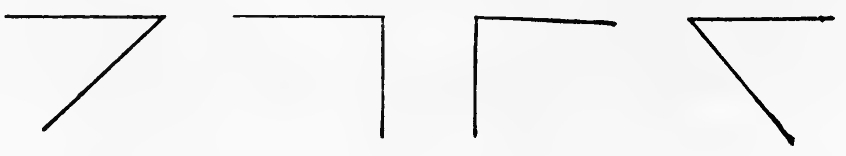

Perform the same operation again for the set,

$\begin{array}{cccc}\mathrm{ba} & \mathrm{ab} & \mathrm{ba} & \mathrm{ab} \\ \mathrm{cb} & \mathrm{cb} & \mathrm{bc} & \mathrm{bc} \\ - & - & - & - \\ \mathrm{ca} & \mathrm{ca} & \mathrm{ca} & \mathrm{ca}\end{array}$


and we should have, allowing the letters this time to be associated with the "figure," a repetition of the same term-orders but differently arranged, viz.,
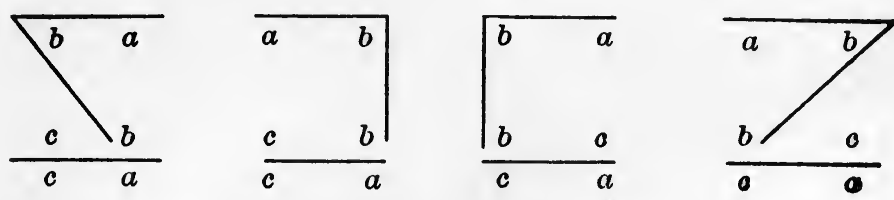

These, accordingly, contain every possible distinction of termorder and will be constantly referred to as the first, second, third and fourth figures of the syllogism respectively. The four figures are easily remembered as combined in a triangle standing on its vertex.

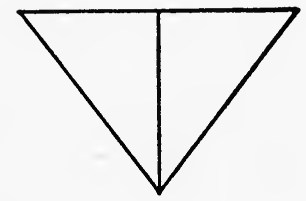

$\S 14$. Let us proceed to summarize our results and to define certain technical expressions. The syllogism is a form of implication belonging to one of the types,
1. $x(b a) y(c b)<z(c a)$
2. $x(a b) y(c b)<z(c a)$
3. $x(b a) y(b c)<z(c a)$
4. $x(a b) y(b c)<z(c a)$

These differences are known as the four figures of the syllogism.' The two forms conjoined in the antecedent are called the premises and the consequent is called the conclusion. The predicate of the conclusion is called the major term and points out the major premise, which by convention is written first in the antecedent. The subject of the conclusion is called the minor term and points out the minor premise. The term which is common to the premises and which does not appear in the conclusion is called the middle term.

If we suppose that $x, y$ and $z$ may take on any one of the

${ }^{1}$ Aristotle recognized only the first three figures of the syllogism. The discovery of the fourth is due to Galen. See Prantl, Geschichte der Logik im Abendlande, Leipzig, 1855, Erster Band, p. 570. 
unprimed letters, $a, \beta, \gamma$ and $\epsilon$, there will be sixty-four syllogistic variations obtained by permuting the four letters three at a time. Each one of these may be expressed in each one of the four figures, so that two hundred and fifty-six cases in all will have to be considered. These are known as the moods of the array. True propositions of the array are known as valid moods of the array. The remainder are known as invalid moods of the array.

In constructing the array of the syllogism, it will prove convenient to omit the implication symbol, $\angle$, and the parts, $(b, a)$, $(c, b)$ and $(c, a)$ and to exhibit each mood as a simple combination of the three letters. If to each one of the sixteen permutations of the four letters taken two at a time, each one of the four letters be added in succession, the array under each figure will appear thus:

$\begin{array}{rrrr}a a a & \beta a a & \gamma a a & \epsilon a a \\ \beta & \beta & \beta & \beta \\ \gamma & \gamma & \gamma & \gamma \\ \epsilon & \epsilon & \epsilon & \epsilon \\ \alpha \beta a & \beta \beta a & \gamma \beta a & \epsilon \beta a \\ \beta & \beta & \beta & \beta \\ \gamma & \gamma & \gamma & \gamma \\ \epsilon & \epsilon & \epsilon & \epsilon \\ a \gamma a & \beta \gamma a & \gamma \gamma a & \epsilon \gamma a \\ \beta & \beta & \beta & \beta \\ \gamma & \gamma & \gamma & \gamma \\ \epsilon & \epsilon & \epsilon & \epsilon \\ a \epsilon a & \beta \epsilon a & \gamma \epsilon a & \epsilon \epsilon a \\ \beta & \beta & \beta & \beta \\ \gamma & \gamma & \gamma & \gamma \\ \epsilon & \epsilon & \epsilon & \epsilon\end{array}$

Exercise.-Construct the array of the syllogism and pick out by the aid of Euler's diagrams the valid and the invalid moods under each figure.

$\S 15$. As was done in the case of immediate inference, we shall now formulate rules for the deduction of the moods of the syllogism. The rules and postulates below will yield all of the valid moods under each figure.

Definition.-Two forms are said to be alike if the one is neither better nor worse than the other. The members of all other pairs are said to be unlike.

Rule 1.-If in any valid mood of the second figure a like minor 
premise and conclusion be each made singly worse, a valid mood will result.

Rule 2.-If in any valid mood of the third figure a like major premise and conclusion be each made singly worse, a valid mood will result.

Rule 3.-If in any valid mood the subject and predicate be interchanged in any form that is simply convertible, a valid mood will result.

Postulate 1. $-a(b a) a(c b) \angle a(c a)$ is a valid mood.

Postulate 2. $-\gamma(b a) \gamma(c b)<\gamma(c a)$ is a valid mood.

Postulate 3. $\epsilon(b a) \gamma(c b)<\epsilon(c a)$ is a valid mood.

Theorems. - The remaining (26) valid moods.

For those who approach the study of the syllogism for the first time it may be well to point out the effect on mood and figure of simple conversion in premise or conclusion.

(a) Simple conversion in the major premise changes the first figure to the second and conversely, the third figure to the fourth and conversely.

(b) Simple conversion in the minor premise changes the first figure to the third and conversely, the second figure to the fourth and conversely.

(c) Simple conversion in the conclusion changes the first figure to the fourth and conversely and leaves the second and third figures unchanged.

It must of course not escape the beginner's notice that the effect of simple conversion in the conclusion is to reverse the normal order of the premises, since the major term becomes the minor term and the minor term becomes the major term.

\section{EXERCISES}

In the exercises below the mood is represented as a simple combination of the three Greek letters and the figure is indicated by a subscript.

(1) From $(\epsilon \gamma \epsilon)_{1}$ and the third rule alone deduce $(\epsilon \gamma \epsilon)_{2},(\gamma \epsilon \epsilon)_{2}$ and $(\gamma \in \epsilon)$.

(2) From $(a a a)_{1},(\gamma \gamma \gamma)_{1}$ and $(a \gamma \gamma)_{1}$ and the third rule deduce all of the remaining valid moods by the aid of the additional principle: If in any valid mood of the first figure a like major premise and conclusion be each made singly worse, a valid mood will result.

(3) Assuming only the first and third rules deduce the remaining valid moods from $(a a a)_{1},(\gamma \gamma \gamma)_{1},(\gamma a \gamma)_{1}$ and $(\epsilon \gamma \epsilon)_{1}$.

The rules and postulates for the deduction of the invalid moods of the syllogism are listed below. In enumerating the postulates, on account of the large number of them, the abbreviation of the exercises above has been employed, the prime over the bracket being understood as standing for the words, "is an invalid mood."

Rule 1.- If in any invalid mood of the second figure a like minor 
premise and conclusion be each made singly better, an invalid mood will result.

Rule 2.-If in any invalid mood of the third figure a like major premise and conclusion be each made singly better, an invalid mood will result.

Rule 3.-If in any invalid mood of the fourth figure premises and conclusion which are all alike, be each made singly worse, an invalid mood will result.

Rule 4.-If in any invalid mood whose premises and conclusion are all alike, the conclusion be made better or worse in any degree, an invalid mood will result.

Rule 5.-If in any invalid mood whose premises and conclusion are all unlike, either premise and the conclusion be interchanged, an invalid mood will result.

Rule 6.- If in any invalid mood the subject and predicate be interchanged in any form that is simply convertible, an invalid mood will result.

Postulates:

\begin{tabular}{|c|c|c|c|}
\hline $\begin{array}{l}(a a \beta)^{\prime}{ }_{1} \\
(a a \gamma)^{\prime}{ }_{1} \\
(a a \epsilon)_{1}^{\prime} \\
(a \beta \gamma)^{\prime}{ }_{1}\end{array}$ & $\begin{array}{l}(a \beta \epsilon)^{\prime}{ }_{1} \\
(a \gamma \gamma)^{\prime}{ }_{3} \\
(a \epsilon \gamma)_{1}{ }_{1} \\
(\beta a \gamma)^{\prime}{ }_{1}\end{array}$ & $\begin{array}{l}(\beta \gamma \gamma)_{3}^{\prime} \\
(\beta \epsilon \gamma)_{1}^{\prime} \\
(\gamma a \gamma)_{2}^{\prime} \\
(\gamma \beta \gamma)_{2}^{\prime}\end{array}$ & $\begin{array}{l}(\gamma \gamma \gamma)_{2}^{\prime}{ }_{2} \\
(\gamma \epsilon \gamma)_{2}^{\prime} \\
(\epsilon a \gamma)_{1}^{\prime} \\
(\epsilon \beta \gamma)_{1}^{\prime}\end{array}$ \\
\hline
\end{tabular}

The effect on mood and figure of interchanging either premise and the conclusion (Rule 5) may be conveniently summarized as follows:

(a) Interchanging major premise and conclusion changes the first figure to the third and conversely and the premises remain in normal order.

(b) Interchanging minor premise and conclusion changes the first figure to the second and conversely and the premises remain in normal order.

(c) Interchanging major premise and conclusion changes the second figure to the third and reverses the normal order of the premises.

(d) Interchanging minor premise and conclusion changes the third figure to the second and reverses the normal order of the premises.

(e) Interchanging either premise and conclusion leaves the fourth figure unchanged and reverses the normal order of the premises.

\section{EXERCISES}

(1) From $(\epsilon \beta \epsilon)^{\prime}{ }_{3}$ alone deduce seventy-eight other invalid moods.

(2) From $(\alpha \beta \epsilon)_{1}^{\prime}$ alone deduce twenty-three other invalid moods.

(3) From $(\alpha \beta \gamma)^{\prime}{ }_{1}$ alone deduce eleven other invalid moods.

(4) Deduce the invalid moods of the first figure which have a $\gamma$-minor premise. 
$\S 16$. As in the case of immediate inference we may formulate rules for the immediate detection of the invalid moods of the syllogism. These are six in number.

Rule 1.-A mood is invalid if the conclusion be unlike two like premises.

Rule 2.-A mood is invalid if the conclusion be unlike the worse of two premises.

Rule 3.-A mood is invalid if each premise be in the $\epsilon$-form.

Rule 4.-A mood is invalid if an $\alpha$ - and a $\gamma$-premise be conjoined in the antecedent and the middle term be undistributed in the major premise.

Rule 5.-A mood is invalid if the middle term be undistributed in each premise.

Rule 6.-A mood is invalid if a term which is distributed in the conclusion be undistributed in the premise.

The rules for the immediate detection of the invalid moods of the syllogism are sufficient, because they declare all the moods not already found to be valid to be invalid. They are all necessary, because we can point to at least one example which falls uniquely under each rule.

\section{EXERCISES}

(1) Construct the array of the syllogism and place after each invalid mood the number of a rule that declares it to be invalid.

(2) Show that it follows from one of the rules alone that two $\beta$-premises do not imply a conclusion.

(3) Show that there are only two moods which illustrate the fourth rule uniquely.

(4) Make a list of examples which fall uniquely under each one of the rules. 


\section{CHAPTER IV}

$\S 17$. The type of implication which is now to be considered is one in which the number of terms is greater than three and, as in the case of the syllogism, the number of premises one less than the number of terms. Accordingly, it will be more convenient to employ in place of the class-symbols, $a, b, c$, etc., the ordinal numbers, $1,2,3$, etc.

The sorites is an implication of the general form:

$$
x(1,2) y(2,3) z(3,4) \ldots u(n-1, n)<w(n 1),
$$

in which $n$ is greater than three and in which we follow the convention of writing first the premise which contains the predicate of the conclusion.

In order to illustrate the manner of constructing a valid mood of the sorites from a chain of valid syllogisms, let us set down the following definition and principles:

Definition.-If $x<y$ is a valid implication then $x$ is said to be a strengthened form of $y$ and $y$ is said to be a weakened form of $x$.

Principle i.-If in any valid mood of syllogism or of sorites a premise be strengthened or a conclusion be weakened, a valid implication will result.

Principle ii.-If in any valid mood of syllogism or of sorites the same factor be conjoined to both antecedent and consequent a valid implication will result.

Consider the chain of valid syllogisms,

$$
\begin{aligned}
& \gamma(21) \gamma(32)<\gamma(31), \\
& \gamma(31) \gamma(43)<\gamma(41), \\
& \gamma(41) \gamma(54)<\gamma(51) .
\end{aligned}
$$

Since the major premise of the last member of the chain is the same as the conclusion of the second member, it may be strengthened (by principle i) to $\gamma(31) \gamma(43)$ and we should have:

$$
\gamma(31) \gamma(43) \gamma(54)<\gamma(51)
$$

The major premise of this result is in turn the same as the conclusion of the first member of the chain and may, accordingly, be strengthened to $\gamma(21) \gamma(32)$. The valid mood of the sorites which is implied by the chain of valid syllogisms is therefore:

$$
\begin{gathered}
\gamma(21) \gamma(32) \gamma(43) \gamma(54)<\gamma(51) \\
26
\end{gathered}
$$


Suppose that we were to begin with the first syllogism of the chain and were to conjoin to both antecedent and consequent the minor premise of the second member of the chain (principle ii), i. e.,

$$
\gamma(21) \gamma(32) \gamma(43)<\gamma(31) \gamma(43)
$$

The consequent of this result being the same as the antecedent of the second syllogism, may be weakened (principle i), to $\gamma(41)$ and we should have:

$$
\gamma(21) \gamma(32) \gamma(43)<\gamma(41) \text {. }
$$

Now conjoin, as before, to antecedent and consequent of this mood of the sorites the minor premise of the last syllogism of the chain (principle ii), i. e.,

$$
\gamma(21) \gamma(32) \gamma(43) \gamma(54) \angle \gamma(41) \gamma(54)
$$

the consequent of which weakens in turn (principle i) to $\gamma(51)$ so that there results

$$
\gamma(21) \gamma(32) \gamma(43) \gamma(54)<\gamma(51),
$$

the same sorites as the one obtained by the process which was first described.

$\S 18$. In general, if the chain of syllogisms,

$$
\begin{aligned}
& x_{1}(1,2) x_{2}(2,3)<x_{3}(31), \\
& x_{3}(31) x_{4}(3,4) \angle x_{5}(41), \\
& x_{5}(41) x_{6}(4,5)<x_{7}(51), \\
& \cdot \cdot \cdot \cdot \cdot \cdot \cdot \cdot \cdot \cdot \\
& x_{2 n-5}(n-1) x_{2 n-4}(n-1, n)<x_{2 n-3}(n 1) .
\end{aligned}
$$

be valid throughout in each of its members, then

$$
x_{1}(1,2) x_{2}(2,3) x_{4}(3,4) \ldots x_{2 n-4}(n-1, n)<x_{2 n-3}(n 1)
$$

is a valid mood of the sorites. Accordingly, it is manifest that a certain number of valid moods of the sorites may be constructed from chains of valid syllogisms. It remains to be proven that the only valid moods of the sorites that exist can be built up from chains of valid syllogisms in the manner described. This proof depends upon the following assumptions:

Principle i. - A valid mood of the sorites will remain valid when as many terms have been identified as we desire.

Principle ii.-An unprimed a-premise, whose subject and predicate are identical, may be suppressed as a unit multiplier.

Principle iii.-A valid mood of the sorites, whose premises and conclusion are all unprimed forms and which has one premise of 
the same form as the conclusion, will remain valid when as many other premises as we desire are put in the $a$-form.

Principle iv. - A valid mood of the sorites, whose premises and conclusion are all unprimed forms and none of whose premises has the same form as the conclusion, will remain valid when as many premises as we desire, but one, are put in the $a$-form.

Theorem i.-There exists no valid mood of the sorites whose premises and conclusion are all unprimed forms and none of whose premises has the same form as the conclusion.

For suppose such a valid mood to exist and put all the premises after the first two in the $a$-form (principle iv). Then by identifying terms in the a-premises so obtained (principle i) and suppressing one premise after another (principle ii), we should in the end come upon an invalid syllogism. Accordingly, the mood of the sorites with which we began, must be invalid as well.

In the general solution which follows, it will be convenient to take the conclusion successively in each one of its four possible forms.

\section{$\S 19$. Conclusion in the a-form.}

At least one of the premises is in the a-form if the mood of the sorites is valid (theorem i). If one of the remaining premises $x(s-1, s)$ be not in the $a$-form, put all of the remaining premises except $x$ in the $a$-form, if they be not already in that form (principle iii). Then by identifying terms (principle i) the mood of the sorites will reduce (principle ii) to an invalid syllogism,

$$
\begin{aligned}
& \quad x(s-1, s) a(s, s+1) \angle a(s+1 s-1) . \\
& \text { or } a(s-2, s-1) x(s-1, s)<a(s s-2) .
\end{aligned}
$$

Consequently, all of the premises are in the $a$-form, if the mood of the sorites is valid and our implication must be,

$$
a(1,2) a(2,3) \ldots a(n-1, n)<a(n 1) \text {, }
$$

which can be constructed from the chain of valid syllogisms,

$$
\begin{aligned}
& a(1,2) a(2,3)<a(31), \\
& a(31) a(3,4)<a(41), \\
& \dot{a} \cdot \dot{1} \dot{1} \dot{a} \cdot \dot{1} \cdot \dot{1})<a(n 1) .
\end{aligned}
$$

$\S 20$. Conclusion in the $\beta$-form.

At least one of the premises is in the $\beta$-form, if the mood is valid (theorem i), and all of the other premises are in the $a$-form. For, 
suppose one of the other premises $x(s-1, s)$ were not in the $a$-form. Put all of the other premises except $x$ and the $\beta$-premise in the $a$-form (principle iii). Then, by identifying terms (principle i), the mood of the sorites will be reducible to an invalid syllogism (principle ii) of the form,

$$
\begin{array}{r}
\beta(s-1, s-2) x(s-1, s)<\beta(s s-2), \\
\text { or } x(s-1, s) \beta(s, s+1)<\beta(s+1 s-1) .
\end{array}
$$

Consequently, the sorites must be

$$
a(1,2) \ldots a(s, s-1) \beta(s, s+1) \ldots a(n-1, n)<\beta(n 1) \text {, }
$$

and this may be built up out of the chain of valid syllogisms,

$$
\begin{aligned}
& a(1,2) a(2,3)<a(31) \text {, } \\
& a(31) a(3,4)<a(41) \text {, } \\
& a(s-11) a(s-1, s)<a(s 1) \text {, } \\
& a(s 1) \beta(s, s+1) \angle \beta(s+11) \text {, } \\
& \beta(s+11) a(s+1, s+2) \angle \beta(s+21) \text {, } \\
& \beta(n-11) a(n-1, n)<\beta(n 1) .
\end{aligned}
$$

$\S 21$. Conclusion in the $\gamma$-form.

At least one of the premises must be in the $\gamma$-form, if the mood of the sorites is to be valid (theorem i). Moreover, each $\gamma$-premise that occurs, must present its terms in the order $(s s-1)$, i. e., with the larger ordinal number coming first. For suppose that $\gamma(s-1 s)$ should appear as one of the premises. By putting each one of the remaining premises in the $a$-form (principle iii) and by identifying terms (principle $i$ ), the sorites will reduce to an invalid syllogism (principle ii),

$$
\begin{aligned}
& \gamma(s-1 s) a(s, s+1)<\gamma(s+1 s-1), \\
& \text { or } a(s-1, s-2) \gamma(s-1 s)<\gamma(s s-2) .
\end{aligned}
$$

Just as in the cases already considered it can be shown that no $\beta$ - or $\epsilon$-premise can occur. One valid mood of the sorites may consequently be

$$
\gamma(21) \gamma(32) \ldots \gamma(n n-1)<\gamma(n 1),
$$

which can, in fact, be constructed from the chain of valid syllogisms,

$$
\begin{aligned}
& \gamma(21) \gamma(32)<\gamma(31), \\
& \gamma(31) \gamma(43)<\gamma(41), \\
& \cdot \dot{\cdot} \cdot \dot{\cdot} \cdot \dot{\cdot} \cdot \dot{1}) \\
& \gamma(n-11) \gamma(n n-1)<\gamma(n 1) .
\end{aligned}
$$


It will now be manifest that all the other forms of valid sorites with a $\gamma$-conclusion are to be gotten from the above type by transforming one or more of the premises into the $a$-form in every possible way under the restrictions of theorem $i$ and it will be easy in each case to construct the chain of generating syllogisms.

\section{§22. Conclusion in the $\epsilon$-form.}

At least one of the premises is in the $\epsilon$-form (theorem i) and there is not more than one $\epsilon$-premise. For, if there were two or more $\epsilon$-premises, we might put all but two of the $\epsilon$-premises in the $a$-form (principle iii). Then, by identifying terms (principle $i$ ) we should come upon an invalid syllogism (principle ii),

$$
\epsilon(s-1, s) \epsilon(s, s+1)<\epsilon(s+1 s-1) .
$$

There can be present no $\beta$-premise. For suppose one or more such premises to be present. By putting all of the premises except one $\beta$-premise and the $\epsilon$-premise in the $a$-form (principle iii), we should by identifying terms (principle i) come upon an invalid syllogism (principle ii),

$$
\begin{array}{r}
\beta(s-1, s) \epsilon(s, s+1)<\epsilon(s+1 s-1), \\
\text { or } \epsilon(s-2, s-1) \beta(s-1, s)<\epsilon(s s-2) .
\end{array}
$$

Any $\gamma$-premise coming after the $\epsilon$-premise must present its terms in the order $(s s-1)$. For suppose $\gamma(s, s-1)$ coming after the $\epsilon$-premise, to present the term-order $(s-1 s)$. Putting all the premises except $\gamma(s-1 s)$ and the $\epsilon$-premise in the $a$-form (principle iii) we should by identifying terms (principle i) come upon an invalid syllogism (principle ii),

$$
\epsilon(s-1, s-2) \gamma(s-1 s)<\epsilon(s s-2) \text {. }
$$

Any $\gamma$-premise coming before the $\epsilon$-premise must present its terms in the order $(s-1 s)$. For suppose $\gamma(s, s-1)$, coming before the $\epsilon$-premise to present the term-order $(s s-1)$. Putting all of the premises except $\gamma(s s-1)$ and the $\epsilon$-premise in the $a$-form (principle iii), we should by identifying terms (principle i) come upon an invalid syllogism (principle ii),

$$
\gamma(s s-1) \epsilon(s, s+1)<\epsilon(s+1 s-1) .
$$

Consequently, one valid mood of the sorites may be $\gamma(12) \ldots \gamma(s-1 s) \epsilon(s, s+1) \gamma(s+2 s+1) \ldots \gamma(n n-1)$ $\angle \epsilon(n 1)$

which can, in fact, be constructed from the chain of valid syllogisms, 


$$
\begin{aligned}
& \gamma(12) \gamma(23)<\gamma(13), \\
& \gamma(13) \gamma(34)<\gamma(14), \\
& \dot{\gamma}(\dot{1}-1) \dot{\gamma}(\dot{s}-1 s) \angle \gamma(1 s), \\
& \gamma(1 s) \epsilon(s, s+1) \angle \epsilon(s+11), \\
& \epsilon(s+11) \gamma(s+2 s+1)<\epsilon(s+21), \\
& \dot{\epsilon}(n-i 1) \dot{\gamma}(n n-1)<\epsilon(n 1) .
\end{aligned}
$$

All the other forms of valid sorites with an f-conclusion are clearly to be gotten from the type that has just been established by putting one or more of the $\gamma$-premises into the $a$-form, and it will be easy in each case to construct the corresponding chain of generating syllogisms.

There exist consequently no valid moods of the sorites, whose premises and conclusion are all unprimed forms, which cannot be built up out of chains of valid syllogisms.

When the general solution of the sorites has been effected by means of the principles of exercise (5) below, i. e., when all valid implications of the form,

$$
x(1,2) y(2,3) \ldots z z(n-1, n)<w(n 1),
$$

have been determined, we shall have resolved at the same time a type of inference composed of the product of $n$ premises, containing a cycle of $n$ terms and implying zero, viz.,

$$
x(1,2) y(2,3) \ldots z(n, 1)<0 .
$$

That the solution of this last type is exactly equivalent to the one given follows from the principles:

$$
\begin{aligned}
& (x<y)<\left(x y^{\prime}<0\right), \\
& \left(x y^{\prime}<0\right)<(x<y) .
\end{aligned}
$$

\section{Exercises}

(1) Construct a valid mood of the sorites from the following chain of valid syllogisms,

$$
\begin{aligned}
& a(21) \gamma(32)<\gamma(31), \\
& \gamma(31) a(43)<\gamma(41), \\
& \gamma(41) \gamma(54)<\gamma(51) .
\end{aligned}
$$

(2) Employing the principles of this chapter, reduce the sorites,

$$
a(21) \gamma(32) a(43) \gamma(54) \angle \gamma(51) \text {, }
$$

successively to each one of the three valid syllogisms of exercise (1).

(3) Employing the principles of this chapter, establish the invalidity of the sorites,

$$
\gamma(21) \gamma(32) \gamma(34) \gamma(54)<\gamma(51) \text {. }
$$


(4) From what chain of valid syllogisms can the sorites,

be built up?

$$
\gamma(12) \gamma(23) \epsilon(34) \gamma(54)<\epsilon(51) \text {, }
$$

(5) Complete the general solution of the sorites begun in this chapter, taking for granted the following principles:

Principle v.-A valid mood of the sorites, whose premises are all unprimed forms and whose conclusion is a primed form and all of whose premises and conclusion are of the same form, will remain valid, when as many premises as we desire, but one, are put in the $a$-form.

Principle vi.-A valid mood of the sorites, whose premises are all unprimed forms and whose conclusion is a primed form and one of whose premises is a form different from the conclusion, will remain valid, when as many other premises as we desire, are put in the $a$-form. 


\section{CHAPTER V}

$\S 23$. In the chapters which follow, the solutions already given will be generalized, new results will appear and the whole will be more completely expressed in the symbolical language of an algebra. ${ }^{1}$

A principle, which is altogether fundamental and which will be taken for granted at each step of our progress, is this: If a proposition is true in general, it is because it remains true for all specific meanings of the terms that enter into it, although an untrue proposition does not always remain untrue in the same circumstances. Thus $\gamma(a b) \angle \gamma(b a)$ is untrue in general, but it becomes true for the case, in which $a$ and $b$ are identical, viz., $\gamma(a a) \angle \gamma(a a)$. Accordingly, when we write $\{x(a, b) \angle y(a, b)\}^{\prime}$, we only assert that there is at least one value of $a$ and one value of $b$ which will render $x(a, b) \angle y(a, b)$ a false proposition. If it has been established that $x(a a) \angle y(a a)$ is untrue, then we may at once infer that the more general implication, $x(a, b) \angle y(a, b)$ is untrue as well. In order to establish the untruth of a given proposition, it will be enough to point to a special instance of its being untrue.

"The Implicative Function is a propositional function with two arguments $p$ and $q$, and is the proposition that either not- $p$ or $q$ is true, that is, it is the proposition $p^{\prime}+q$. Thus if $p$ is true, $p^{\prime}$ is false, and accordingly the only alternative left by the proposition $p^{\prime}+q$ is that $q$ is true. In this sense the proposition $p^{\prime}+q$ will be quoted as stating that $p$ implies q." (Whitehead and Russell, Principia Mathematica, Cambridge University Press, 1910, vol. 1, p. 7.) This limitation we do not allow, for it will be found to run counter to the whole meaning of the system we are building up. In the citation above we have replaced the authors' notation by our own.

\$24. In presenting the materials of our subject-matter we shall have to deal with two types of proposition. The truth of

$$
(x<y)(y<z)<(x<z)
$$

is independent of $x, y$ and $z$, no matter for what propositions

${ }^{1}$ Symbolic logic was first developed by Boole in a work entitled $A n$ Investigation of the Laws of Thought, 1854. Leibnitz (1646-1716) in his youth had suggested the plan of such a calculus but he never executed it. Toward the end of his life he remarked that in such a universal language the characters or symbols would direct the operations of thought and all error would be reduced to error of computation. 
$x, y$ and $z$ may stand. Such a general truth will be termed a principle. The truth of

$$
\gamma(b a) \gamma(c b)<\gamma(c a)
$$

is independent of $a, b$ and $c$, no matter for what classes, $a, b$ and $c$ may stand. If such a general truth has to be taken for granted, it will be termed a postulate.

Principles are, accordingly, independent of forms; postulates are independent of terms.

$\$ 25$. We begin by setting down five postulates, the truth of which should be verified again empirically by the familiar device of Euler's circular diagrams.

$$
\begin{array}{ll}
\text { (i) } & a(b a) a(b c)<a(c a), \\
\text { (ii) } a(b a) \beta(b c)<\beta(c a), & < \\
\text { (iii) } a(b a) \gamma(c b)<\gamma(c a), \\
\text { (iv) } a(b a) \epsilon(b c)<\epsilon(c a), \\
\text { (v) } \gamma(b a) a(b c)<\gamma(c a),
\end{array}
$$

and we shall add to these

(vi) $a^{\prime}(a a)<a(a a)$.

This last assumption illustrates an extension of the common meaning of implication and is forced upon us, if we are to allow $a(a a)$ to stand for a true proposition. The uses, to which such an extension of meaning may be put, will become clear in the sequel. It will be enough to state that a proposition, which is true for all meanings of the terms, will be implied by the proposition whose symbol is $i$, and behaves like a unit multiplier in this algebra.

Only a small number of the principles, which we shall introduce as necessity requires, are independent, but it will not concern our purpose to point out the manner of their inter-connection.

From the principle,

we obtain, by (vi), the theorem,

$$
\left(x^{\prime}<x\right)<(y<x),
$$

$$
i<a(a a) \text {. }
$$

By (i), for $a=b, a(a a) a(a c) \angle a(c a)$, and

$$
\{a(a a) a(a c)<a(c a)\}\{i \angle a(a a)\}<\{a(a c) \angle a(c a)\} \text {, }
$$

by $(x y<z)(w<x)<(w y<z)$,

omitting the unit multiplier, $i$, as it is the custom to do. Similarly, we obtain

$$
\begin{aligned}
& \beta(a c)<\beta(c a), \text { from (ii), } \\
& \gamma(c a)<\gamma(c a), \text { from (iii), } \\
& \epsilon(a c)<\epsilon(c a), \text { from (iv). }
\end{aligned}
$$


Again, $\{a(a b)<a(b a)\}\{a(b a)<a(a b)\}<\{a(a b)<a(a b)\}$. by $(x<y)(y<z)<(x<z)$.

Accordingly,

$$
\begin{aligned}
& a(a b)<a(a b), \\
& \beta(a b)<\beta(a b), \\
& \gamma(a b)<\gamma(a b), \\
& \epsilon(a b)<\epsilon(a b) .
\end{aligned}
$$

A complete induction of the members of this set and an application of the principle,

$$
(x<y)<\left(y^{\prime}<x^{\prime}\right)
$$

yields the general result,

$$
\text { I. } \mathbf{k}^{\prime}(a b) \angle \mathbf{k}^{\prime}(a b) \text {, }
$$

$\mathbf{k}(a b)$ being understood as representing any one of the unprimed letters, $a, \beta, \gamma, \epsilon$.

\$26. Each one of the propositions so far derived, being true, is implied by the unit multiplier, $i$. The contradictory (as it is called) of $i$ will be any proposition, which is untrue for all meanings of the terms that enter into it. It will be represented by the symbol, 0 , and will be defined by

$$
0<i, \quad(i<0)^{\prime} \text {, }
$$

wherein it will be seen that $i$ stands for $o^{\prime}$. For a verbal interpretation of $o$ and $i$ we may read:

$$
\begin{aligned}
& o=\text { No proposition is true, } \\
& i=\text { One proposition is true. }
\end{aligned}
$$

$\S 27$. The utility of the concept of zero, $o$ (the null-proposition), and of one, $i$ (the one-proposition), will be illustrated in part by the derivations, which follow. We have

$$
\{a(a b)<a(a b)\}<\left\{a(a b) a^{\prime}(a b)<o\right\},
$$

by $(x<y)<\left(x y^{\prime}<0\right)$;

$$
\left\{a(a b) a^{\prime}(a b)<0\right\}<\left\{a(a b)<a^{\prime \prime}(a b)\right\},
$$

by $(x y<0)<\left(x<y^{\prime}\right)$.

Thus we should obtain

$$
\begin{aligned}
& a(a b)<a^{\prime \prime}(a b), \\
& \beta(a b)<\beta^{\prime \prime}(a b), \\
& \gamma(a b)<\gamma^{\prime \prime}(a b), \\
& \epsilon(a b)<\epsilon^{\prime \prime}(a b) .
\end{aligned}
$$


by $(x<y)<\left(y^{\prime}<x^{\prime}\right)$;

$$
\{a(a b)<a(a b)\}<\left\{a^{\prime}(a b)<a^{\prime}(a b)\right\},
$$

$$
\left\{a^{\prime}(a b)<a^{\prime}(a b)\right\}<\left\{a^{\prime \prime}(a b)<a(a b)\right\},
$$

by $\left(x^{\prime} \angle y\right)<\left(y^{\prime} \angle x\right)$.

Accordingly,

$$
\begin{aligned}
& a^{\prime \prime}(a b)<a(a b), \\
& \beta^{\prime \prime}(a b)<\beta(a b), \\
& \gamma^{\prime \prime}(a b)<\gamma(a b), \\
& \epsilon^{\prime \prime}(a b)<\epsilon(a b),
\end{aligned}
$$

and a general result will be obvious, viz.,

II. $\mathrm{k}(a b) \angle \mathrm{k}^{\prime \prime}(a b), \quad \mathrm{k}^{\prime \prime}(a b) \angle \mathrm{k}(a b)$, wherein the same restriction is imposed upon $\mathrm{k}(a b)$ as before.

$\S 28$. The principle that the truth of any one of the four categorical forms implies the falsity of each one of the others, a generalization which will now be established, is characteristic of the logic which we are constructing. We shall begin by setting down the three characteristic postulates,

Then, by the principle,

$$
\begin{array}{ll}
\text { (vii) } & \beta(a a)<\beta^{\prime}(a a), \\
\text { (viii) } & \gamma(a a)<\gamma^{\prime}(a a), \\
\text { (ix) } \quad \epsilon(a a) & <\epsilon^{\prime}(a a) .
\end{array}
$$

we may establish at once

$$
\left(x<x^{\prime}\right)<(x<y)
$$

$$
\begin{aligned}
& \beta(a a)<o, \\
& \gamma(a a)<o, \\
& \epsilon(a a)<o .
\end{aligned}
$$

Postulate (ii) above yields, for $a=c, a(b a) \beta(b a) \angle \beta(a a)$; $\{a(b a) \beta(b a) \angle \beta(a a)\}\{\beta(a a) \angle o\} \angle\{a(b a) \beta(b a) \angle o\}$, by $(x<y)(y<z)<(x<z)$;

$$
\{a(b a) \beta(b a)<o\}<\left\{a(b a) \angle \beta^{\prime}(b a)\right\} \text {, }
$$

by $(x y<0)<\left(x<y^{\prime}\right)$.

Similarly, by (iii), $a(b a) \angle \gamma^{\prime}(a b)$;

$$
\{a(a b)<a(b a)\}\left\{a(b a) \angle \gamma^{\prime}(a b)\right\}<\left\{a(a b) \angle \gamma^{\prime}(a b)\right\}
$$

by $(x<y)(y<z)<(x<z)$;

and, by (iv), we obtain $a(a b) \angle \epsilon^{\prime}(a b)$.

If now we postulate,

$$
\begin{aligned}
& \text { (x) } \gamma(b a) \gamma(c b)<\gamma(c a) \text {, } \\
& \text { (xi) } \epsilon(b a) \gamma(c b)<\epsilon(c a) \text {, }
\end{aligned}
$$


there result as before $\epsilon(a b) \angle \gamma^{\prime}(a b)$ and $\gamma(a b) \angle \gamma^{\prime}(b a)$, and if

(xii) $\beta(a b) \angle \gamma^{\prime}(a b)$,

(xiii) $\beta(a b)<\epsilon^{\prime}(a b)$,

all of the remaining implications of this form, viz.,

$$
\begin{array}{ll}
\epsilon(a b)<a^{\prime}(a b) & \gamma(a b)<a^{\prime}(a b) \\
\epsilon(a b)<\beta^{\prime}(a b) & \gamma(a b)<\beta^{\prime}(a b) \\
\epsilon(a b)<\gamma^{\prime}(a b) & \beta(a b)<a^{\prime}(a b)
\end{array}
$$

are obtained at once from those that have just been established by

$$
\left(x<y^{\prime}\right)<\left(y<x^{\prime}\right) \text {. }
$$

If, now, $\mathrm{k}(a b)$ and $\mathrm{w}(a b)$ can not represent the same categorical form, $\gamma(a b)$ and $\gamma(b a)$ being considered distinct, and if further $\mathrm{k}(a b)$ and $\mathrm{w}(a b)$ can stand only for the unprimed letters, $a, \beta, \gamma$ and $\epsilon$, then,

$$
\text { III. } \mathrm{k}(a b)<\mathrm{w}^{\prime}(a b) \text {. }
$$

$\S 29$. We shall now establish the untruth of certain forms of implication, making them ultimately depend upon the invalidity of $i \angle 0$, whose untruth is set down as a matter of definition. ${ }^{2}$

Suppose $a(a a) \angle a^{\prime}(a a)$ were true.

$$
\{i<a(a a)\}\left\{a(a a) \angle a^{\prime}(a a)\right\}<\left\{i \angle a^{\prime}(a a\},\right.
$$

by $(x<y)(y<z)<(x<z)$;

by the same principle.

$$
\left\{i \angle a^{\prime}(a a)\right\}\left\{a^{\prime}(a a)<o\right\}<\{i \angle o\},
$$

But $i \angle O$ is untrue.

$\therefore a(a a) \angle a^{\prime}(a a)$ is untrue as well.

Again,

$$
\left\{a(a a)<a^{\prime}(a a)\right\}^{\prime}\left\{\beta(a a)<a^{\prime}(a a)\right\}<\{a(a a)<\beta(a a)\}^{\prime},
$$

by $(x<z)^{\prime}(y<z)<(x<y)^{\prime}$;

$$
\left\{a(a a) \angle \beta^{\prime}(a a)\right\}\left\{a ( a a ) \angle \beta ( a a \} ^ { \prime } \angle \left\{\beta^{\prime}(a a) \angle \beta(a a\}^{\prime},\right.\right.
$$
by $(x<y)(x<z)^{\prime} \angle(y<z)^{\prime}$.

Accordingly, we have

$$
\begin{aligned}
& \left\{a(a a)<a^{\prime}(a a)\right\}^{\prime}, \\
& \left\{\beta^{\prime}(a a)<\beta(a a)\right\}^{\prime}, \\
& \left\{\gamma^{\prime}(a a)<\gamma(a a)\right\}^{\prime}, \\
& \left\{\epsilon^{\prime}(a a)<\epsilon(a a)\right\}^{\prime},
\end{aligned}
$$

and, since the untruth of any proposition is implied, whenever we can point to a special instance of its being untrue, it follows that

\footnotetext{
${ }^{2}$ Some of the possibilities of the method of reductio ad absurdum in logic were pointed out and elaborated by the famous Italian geometer Saccheri (Logica demonstrativa, 1697).
} 


$$
\begin{aligned}
& \left\{a(a b)<a^{\prime}(a b)\right\}^{\prime}, \\
& \left\{\beta^{\prime}(a b)<\beta(a b)\right\}^{\prime}, \\
& \left\{\gamma^{\prime}(a b)<\gamma(a b)\right\} \\
& \left\{\epsilon^{\prime}(a b)<\epsilon(a b)\right\}^{\prime} .
\end{aligned}
$$

The first member of the set

$$
\begin{aligned}
& \left\{a^{\prime}(a b)<a(a b)\right\}^{\prime}, \\
& \left.\left\{\beta(a b)<\beta^{\prime}(a b)\right\}\right\}^{\prime}, \\
& \left.\left\{\gamma(a b)<\gamma^{\prime}(a b)\right\}\right\}^{\prime}, \\
& \left.\left\{\epsilon(a b)<\epsilon^{\prime}(a b)\right\}\right\}^{\prime},
\end{aligned}
$$

may be gotten from one of the other three, thus,

$$
\left\{\gamma(a b)<\gamma^{\prime}(a b)\right\}^{\prime}\left\{a(a b)<\gamma^{\prime}(a b)\right\}<\{\gamma(a b)<a(a b)\}^{\prime} \text {, }
$$
by $(x<z)^{\prime}(y<z)<(x<y)^{\prime}$;

$$
\left\{\gamma(a b)<a^{\prime}(a b)\right\}\{\gamma(a b)<a(a b)\}^{\prime}<\left\{a^{\prime}(a b)<a(a b)\right\}^{\prime} \text {. }
$$
by $(x<y)(x<z)^{\prime} \angle(y<z)^{\prime}$.

For the reduction of the last three see exercise (10) at the end of this chapter.

As a result of a complete induction of the members of these sets and upon application of $(x<y)^{\prime} \angle\left(y^{\prime}<x^{\prime}\right)^{\prime}$, it follows that

$$
\begin{aligned}
& \text { IV. }\left\{\mathrm{k}(a b) \angle \mathrm{k}^{\prime}(a b)\right\}^{\prime}, \quad\left\{\mathrm{k}^{\prime}(a b)<\mathrm{k}(a b)\right\}^{\prime} \text {, } \\
& \left\{\mathrm{k}^{\prime}(a b) \angle \mathrm{k}^{\prime \prime}(a b)\right\}^{\prime}, \quad\left\{\mathrm{k}^{\prime \prime}(a b)<\mathrm{k}^{\prime}(a b)\right\}^{\prime} \text {. }
\end{aligned}
$$

$\S 30$. If $a^{\prime}(a b) \angle \beta(a b)$ were a true implication, we should have:

$$
\left\{\gamma(a b)<a^{\prime}(a b)\right\}\left\{a^{\prime}(a b) \angle \beta(a b)\right\}<\{\gamma(a b) \angle \beta(a b)\},
$$
by $(x<y)(y<z)<(x<z)$;

$$
\{\gamma(a b)<\beta(a b)\}\left\{\beta(a b)<\gamma^{\prime}(a b)\right\}<\left\{\gamma(a b)<\gamma^{\prime}(a b)\right\}
$$
by the same principle.

$$
\therefore a^{\prime}(a b) \angle \beta(a b) \text { is untrue. }
$$

Applying the same method of reduction there will result:

and upon application of

$$
\begin{array}{cc}
\left\{a^{\prime}(a b)<\beta(a b)\right\}^{\prime}, & \left\{\beta^{\prime}(a b)<\gamma(a b)\right\}^{\prime}, \\
\left\{a^{\prime}(a b)<\gamma(a b)\right\}^{\prime}, & \left\{\beta^{\prime}(a b)<\epsilon(a b)\right\}^{\prime}, \\
\left\{a^{\prime}(a b)<\epsilon(a b)\right\}^{\prime}, & \left\{\gamma^{\prime}(a b)<\epsilon(a b)\right\}^{\prime} . \\
\left\{\gamma^{\prime}(a b)<\gamma(b a)\right\}^{\prime}, &
\end{array}
$$

$$
\begin{aligned}
& \left(x^{\prime}<y\right)^{\prime}<\left(y^{\prime} \angle x\right)^{\prime}, \\
\left\{\epsilon^{\prime}(a b)<a(a b)\right\}^{\prime}, & \left\{\gamma^{\prime}(a b)<a(a b)\right\}^{\prime}, \\
\left\{\epsilon^{\prime}(a b)<\beta(a b)\right\}^{\prime}, & \left\{\gamma^{\prime}(a b)<\beta(a b)\right\}^{\prime}, \\
\left\{\epsilon^{\prime}(a b)<\gamma(a b)\right\}^{\prime}, & \left\{\beta^{\prime}(a b)<a(a b)\right\}^{\prime} .
\end{aligned}
$$

We are now prepared to lay down the final generalizations which 
are given below. From the propositions that have just been enumerated there will follow

from III and V, by

$$
\text { V. }\left\{\mathbf{w}^{\prime}(a b)<\mathbf{k}(a b)\right\}^{\prime} \text {; }
$$

$$
\begin{aligned}
& (x<y)<\left(y^{\prime}<x^{\prime}\right) \\
& (x<y)^{\prime}<\left(y^{\prime}<x^{\prime}\right)^{\prime}
\end{aligned}
$$

VI. $\quad \mathrm{k}^{\prime \prime}(a b) \angle \mathrm{w}^{\prime}(a b), \quad\left\{\mathrm{w}^{\prime}(a b) \angle \mathrm{k}^{\prime \prime}(a b)\right\}^{\prime}$;

from III and IV, by

$$
\begin{gathered}
(x<z)^{\prime}(y<z)<(x<y)^{\prime}, \\
(x<y)^{\prime}<\left(y^{\prime}<x^{\prime}\right)^{\prime},
\end{gathered}
$$

VII. $\left\{\mathrm{k}(a b) \angle \mathrm{w}^{\prime \prime}(a b)\right\}^{\prime}, \quad\left\{\mathrm{w}^{\prime \prime}(a b) \angle \mathrm{k}(a b)\right\}^{\prime}$.

$\S 31$. In order to classify the categorical forms under the heads, contradictories, contraries, subcontraries, and subalterns, let us consider what special meanings of $x(a b)$ and $y(a b)$ render true or untrue,

$$
\begin{aligned}
& \text { (1) } x(a b)<y^{\prime}(a b), \\
& \text { (2) } y^{\prime}(a b)<x(a b) \text {. }
\end{aligned}
$$

If $x(a b)$ and $y(a b)$ satisfy (1) and (2) together, $x(a b)$ is said to be contradictory to $y(a b)$. By $\mathrm{I}, \mathrm{k}^{\prime}(a b)$ is contradictory to $\mathrm{k}(a b)$ and, by II, $\mathrm{k}(a b)$ is contradictory to $\mathrm{k}^{\prime}(a b)$.

If $x(a b)$ and $y(a b)$ satisfy (1) alone, $x(a b)$ is said to be contrary to $y(a b)$. By III and V, $\mathrm{k}(a b)$ is contrary to $\mathrm{w}(a b)$.

If $x(a b)$ and $y(a b)$ satisfy (2) alone $x(a b)$ is said to be subcontrary to $y(a b)$. By VI, $\mathrm{k}^{\prime}(a b)$ is subcontrary to $\mathrm{w}^{\prime}(a b)$.

If $x(a b)$ and $y(a b)$ satisfy neither (1) nor (2), $x(a b)$ is said to be subaltern to $y(a b)$. By VII, $\mathrm{k}(a b)$ is subaltern to $\mathrm{w}^{\prime}(a b)$, and, by IV, $\mathrm{k}(a b)$ and $\mathrm{k}^{\prime}(a b)$ are each the subalterns of themselves.

\section{EXERCISES}

1. The meaning of logical equality is given by

$$
\begin{aligned}
& (x<y)(y<x)<(x=y), \\
& (x=y) \angle(x<y)(y<x) .
\end{aligned}
$$

If $\mathrm{k}(a b)=\mathrm{k}(a b) \mathrm{k}(a b)$ and $\mathrm{k}(a b) \angle \mathrm{w}^{\prime}(a b)$, show that

$$
\begin{aligned}
& a(a b) \angle \beta^{\prime}(a b) \gamma^{\prime}(a b) \epsilon^{\prime}(a b) \gamma^{\prime}(b a), \\
& \beta(a b)<a^{\prime}(a b) \gamma^{\prime}(a b) \epsilon^{\prime}(a b) \gamma^{\prime}(b a), \\
& \gamma(a b)<a^{\prime}(a b) \beta^{\prime}(a b) \epsilon^{\prime}(a b) \gamma^{\prime}(b a), \\
& \epsilon(a b)<a^{\prime}(a b) \beta^{\prime}(a b) \gamma^{\prime}(a b) \gamma^{\prime}(b a),
\end{aligned}
$$

by the aid of

$$
\begin{aligned}
& (x<y)(y<z)<(x<z), \\
& (x<y)<(z x<z y) .
\end{aligned}
$$


2. If

establish

$$
\begin{aligned}
& \beta^{\prime}(a b) \gamma^{\prime}(a b) \epsilon^{\prime}(a b) \gamma^{\prime}(b a)<a(a b), \\
& a^{\prime}(a b) \gamma^{\prime}(a b) \epsilon^{\prime}(a b) \gamma^{\prime}(b a)<\beta(a b), \\
& a^{\prime}(a b) \beta^{\prime}(a b) \epsilon^{\prime}(a b) \gamma^{\prime}(b a)<\gamma(a b), \\
& a^{\prime}(a b) \beta^{\prime}(a b) \gamma^{\prime}(a b) \gamma^{\prime}(b a)<\epsilon(a b),
\end{aligned}
$$

$$
\begin{aligned}
& a^{\prime}(a b)=\beta(a b)+\gamma(a b)+\epsilon(a b)+\gamma(b a), \\
& \beta^{\prime}(a b)=a(a b)+\gamma(a b)+\epsilon(a b)+\gamma(b a), \\
& \gamma^{\prime}(a b)=a(a b)+\beta(a b)+\epsilon(a b)+\gamma(b a), \\
& \epsilon^{\prime}(a b)=a(a b)+\beta(a b)+\gamma(a b)+\gamma(b a),
\end{aligned}
$$

assuming that the contradictory of a product is the sum of the contradictories of the separate factors and assuming the right to substitute $\mathrm{k}(a b)$ directly for $\mathrm{k}^{\prime \prime}(a b)$.

3. Assuming $x(a b)=x(a b) x(a b), x(a b)=x(a b)+x(a b)$, show that

$$
\begin{gathered}
a^{\prime}(a b) \beta^{\prime}(a b)=\gamma(a b)+\epsilon(a b)+\gamma(b a), \text { etc., etc., } \\
a(a b)+\beta(a b)=\gamma^{\prime}(a b) \epsilon^{\prime}(a b) \gamma^{\prime}(b a), \text { etc., etc. }
\end{gathered}
$$

4. Establish the general results,

$$
\begin{gathered}
\mathrm{k}(a b)=\mathrm{k}(a b) \mathrm{w}^{\prime}(a b), \quad \mathrm{k}^{\prime}(a b)=\mathrm{k}^{\prime}(a b)+\mathrm{w}(a b), \\
\mathrm{k}(a b) \mathrm{w}(a b)=0 .
\end{gathered}
$$

5. From the principle, $(x<z)^{\prime}(y<z) \angle(x<y)^{\prime}$, and the postulate, $\left\{a(a a) \angle a^{\prime}(a a)\right\}^{\prime}$, derive $\{a(a a) \angle \beta(a a)\}^{\prime},\{a(a a) \angle \gamma(a a)\}^{\prime},\{a(a a)$ $\angle \epsilon(a a)\}^{\prime}$.

6. By the aid of the principles,

$$
(x<y)(y<z)<(x<z), \quad\left(x<x^{\prime}\right)<(x<y),
$$

from the postulate, $a^{\prime}(a a) \angle a(a a)$, and results already established, (viz., III), show that all propositions of the form, $x(a a) \angle y(a a)$, except the three cases in the last example, are true implications, $x(a a)$ and $y(a a)$ representing only the unprimed letters.

7 Show by the method of the last example that $a(a a) \angle a^{\prime}(a a)$ is the only untrue implication of the form $x(a a) \angle y^{\prime}(a a)$.

8. Derive seven true implications of the form, $x^{\prime}(a a) \angle y(a a)$, and nine untrue implications of the same form.

9. Establish the untruth of

$$
\mathrm{k}(a, b) \angle \mathrm{w}(a, b), \quad \mathrm{k}^{\prime}(a, b) \angle \mathrm{w}^{\prime}(a, b) .
$$

10. Establish the untruth of $\beta(a b) \angle \beta^{\prime}(a b)$ and $\epsilon(a b) \angle \epsilon^{\prime}(a b)$, by making them depend upon the untruth of $\beta(b a) \beta(c b) \angle \beta^{\prime}(c a)$ and $\epsilon(b a) \epsilon(c b) \angle \epsilon^{\prime}(c a)$ respectively (see the postulates of the next chapter). Thus,

by $\left.\left(x<y^{\prime}\right)^{\prime} \angle(x y) \beta(c b) \angle o\right)^{\prime}$;

$$
\left\{\beta(b a) \beta(c b)<\beta^{\prime}(c a)\right\}^{\prime} \angle\{\beta(b a) \beta(c b) \beta(c a)<o\}^{\prime},
$$

$\{\beta(b a) \beta(c b) \beta(c a)<o\}^{\prime}\{\beta(c b) \beta(c a) o<0\} \angle$

$$
\left\{\beta(b a) \beta(c b) \beta(c a)<\beta(c b) \beta(c a)_{0}\right\}^{\prime},
$$

by $(x y<z)^{\prime}(w<z) \angle(x y<w)^{\prime}$;

$$
\{\beta(b a) \beta(c b) \beta(c a) \angle \beta(c b) \beta(c a) 0\}^{\prime} \angle\{\beta(b a)<0\}^{\prime} \text {, }
$$

by $(x z<z y)^{\prime} \angle(x<y)^{\prime}$;

by $(x<0)^{\prime} \angle\left(x<x^{\prime}\right)^{\prime}$.

$$
\{\beta(b a)<o\}^{\prime} \angle\left\{\beta(b a)<\beta^{\prime}(b a)\right\}^{\prime} \text {, }
$$




\section{CHAPTER VI}

§32. The valid moods of the syllogism,

$$
x(a, b) y(b, c)<z(c a),
$$

twenty-nine in all, which are not set down among the assumptions of the last chapter, may be derived at once by the following principles:

$$
\begin{aligned}
& (x y<z)(w \angle x)<(w y<z), \\
& (x<y)(y<z)<(x<z), \\
& (x y<z)<(y x<z) .
\end{aligned}
$$

Thus, from postulate (xi), Chap. V, by the second principle,

$$
\{\epsilon(b a) \gamma(c b)<\epsilon(c a)\}\{\epsilon(c a)<\epsilon(a c)\}<\{\epsilon(b a) \gamma(c b)<\epsilon(a c)\} \text {, }
$$

and, since the term-order in the conclusion is now reversed, so that the major term has become the minor term and the minor term has become the major term, it will be necessary to employ the third principle to restore the normal order of the premises. Accordingly,

$$
\{\epsilon(b a) \gamma(c b)<\epsilon(a c)\}<\{\gamma(c b) \epsilon(b a)<\epsilon(a c)\},
$$

and it will be seen that the term order in this result is that of the fourth figure. The second principle (above) thus enables us to convert simply in the conclusion and the effect of simple conversion in the conclusion is to change the first figure to the fourth.

Similarly, since the third principle enables us to arrange the premises in either order, the first principle will allow us to convert simply in either premise, if that premise be not in the $\gamma$-form. Thus, from postulate (i) of the last chapter

$$
\{a(b a) a(b c)<a(c a)\}<\{a(b c) a(b a)<a(c a)\},
$$

by the third principle (above);

$$
\{a(b c) a(b a)<a(c a)\}\{a(c b)<a(b c)\}<\{a(c b) a(b a)<a(c a)\},
$$

by the first principle (above);

$$
\{a(c b) a(b a)<a(c a)\}<\{a(b a) a(c b)<a(c a)\} \text {, }
$$

by the third principle (above); and this result is a valid mood of the first figure. However, when it is desired to convert simply in the minor premise, it will be more convenient to employ at once the principle,

$$
(x y<z)(w<y)<(x w<z),
$$

and avoid two of the three steps, that would otherwise be necessary. 


\section{EXERCISE}

From postulates (i-v) and postulate (xi) of the last chapter derive twenty-two valid moods of the syllogism by the aid of the principles.

$$
\begin{gathered}
(x y<z)(w<x)<(w y<z), \\
(x y<z)(w<y)<(x w<z), \\
(x y<z)(z<w)<(x y<w), \\
(x y<z)<(y x<z) .
\end{gathered}
$$

\$33. The valid moods of the syllogism,

$$
x(a, b) y(b, c)<z^{\prime}(c a),
$$

one hundred and forty-two in number, as well as those of the syllogisms, $x(a, b) y^{\prime}(b, c)<z^{\prime}(c a)$ and $x^{\prime}(a, b) y(b, c)<z^{\prime}(c a)$, which number thirty-one and twenty-seven respectively, may now be obtained from the results of $\$ 32$ and the forms of immediate inference by the aid of the additional principles,

$$
\begin{aligned}
& \left(x y<z^{\prime}\right)<\left(x z<y^{\prime}\right), \\
& \left(x y<z^{\prime}\right)<\left(z y<x^{\prime}\right) .
\end{aligned}
$$

The examples which follow will be enough to illustrate the method.

(1) $\{y(b a) \gamma(c b)<\gamma(c a)\}<\left\{\gamma(b a) \gamma^{\prime}(c a) \angle \gamma^{\prime}(c b)\right\}$

by $(x y<z)<\left(x z^{\prime}<y^{\prime}\right)$.

(2) $\left\{\gamma(a b) \gamma^{\prime}(c b)<\gamma^{\prime}(c a)\right\}\left\{\epsilon(c b) \angle \gamma^{\prime}(c b)\right\}$

by $(x y<z)(w<y)<(x w<z)$.

$$
\angle\left\{\gamma(a b) \epsilon(c b)<\gamma^{\prime}(c a)\right\} \text {, }
$$

(3) $\left\{\gamma(a b) \epsilon(c b)<\gamma^{\prime}(c a)\right\}<\left\{\gamma(a b) \gamma(c a) \angle \epsilon^{\prime}(c b)\right\}$, by $\left(x y<z^{\prime}\right) \angle\left(x z<y^{\prime}\right)$.

No other valid moods of syllogistic form exist, except those that have now been enumerated, as will appear in the sequel, when all of the remaining variants shall have been declared untrue.

\section{EXERCISES}

1. From postulate (xi) deduce six valid implications of the form, $x(a, b) y^{\prime}(b, c)<z^{\prime}(c a)$.

2. From postulate (xi) deduce thirty-three valid implications of the form, $x(a, b) y(b, c)<z^{\prime}(c a)$.

$\S 34$. It will be convenient in establishing the invalid moods of the syllogism to begin with the form

$$
x(a, b) y(b, c)<z^{\prime}(c a) .
$$

Any invalid mood under this head, which contains an $a$-premise or an a-conclusion, may be shown to be invalid by identifying terms in the $a$-form. Thus: 
1. Suppose $a(b a) \gamma(c b) \angle \gamma^{\prime}(c a)$ were valid, and identify terms in the major premise.

$$
\left\{a(a a) \gamma(c a) \angle \gamma^{\prime}(c a)\right\}\{i \angle a(a a)\}<\left\{\gamma(c a) \angle \gamma^{\prime}(c a)\right\} \text {, }
$$
by $(x y \angle z)(w \angle x) \angle(w y<z)$.

$$
\therefore a(b a) \gamma(c b)<\gamma^{\prime}(c a) \text { is invalid. }
$$

2. Suppose $\gamma(a b) \gamma(c b)<a^{\prime}(c a)$ were valid and identify terms in the conclusion.

$$
\left\{\gamma(a b) \gamma(a b)<a^{\prime}(a a)\right\}\left\{a^{\prime}(a a)<o\right\}<\{\gamma(a b) \gamma(a b)<o\},
$$
by $(x y<z)(z<w)<(x y<w)$;

$$
\{\gamma(a b) \gamma(a b)<0\}<\left\{\gamma(a b)<\gamma^{\prime}(a b)\right\} \text {, }
$$

by $(x y<0)<\left(x<y^{\prime}\right)$.

$$
\therefore \gamma(a b) \gamma(c b)<a^{\prime}(c a) \text { is invalid. }
$$

\section{EXERCISE}

Establish the invalidity of the thirty-four moods of the syllogism, $x(a, b) y(b, c)<z^{\prime}(c a)$, which are invalid and which contain an a-premise or an $\boldsymbol{a}^{\prime}$-conclusion.

In order to deduce the invalid moods, which remain, eighty in all, it will be necessary to add eleven postulates to the ones already set down. These assumptions are:

$\beta(b a) \beta(c b) \angle \beta^{\prime}(c a)$ is an invalid mood.
$\beta(b a) \beta(c b) \angle \gamma^{\prime}(c a)$ is an invalid mood.
$\beta(b a) \beta(c b) \angle \epsilon^{\prime}(c a)$ is an invalid mood.
$\gamma(a b) \gamma(c b) \angle \beta^{\prime}(c a)$ is an invalid mood.
$\gamma(b a) \gamma(b c) \angle \beta^{\prime}(c a)$ is an invalid mood.
$\gamma(b a) \gamma(c b) \angle \gamma^{\prime}(c a)$ is an invalid mood.
$\gamma(a b) \gamma(c b) \angle \epsilon^{\prime}(c a)$ is an invalid mood.
$\epsilon(b a) \gamma(b c) \angle \beta^{\prime}(c a)$ is an invalid mood.
$\epsilon(b a) \gamma(b c) \angle \epsilon^{\prime}(c a)$ is an invalid mood.
$\epsilon(b a) \epsilon(c b)<\beta^{\prime}(c a)$ is an invalid mood.
$\epsilon(b a) \epsilon(c b)<\epsilon^{\prime}(c a)$ is an invalid mood.

\section{EXERCISE}

From "the"postulates just set down deduce sixty-nine other non-implications of the same form, by the aid of the additional principles.

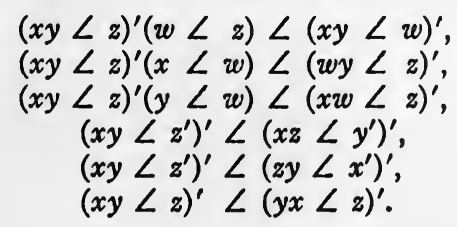


$\S 35$. All of the invalid moods of the syllogistic form, $x(a, b)$ $y(b, c)<z(c a), x^{\prime}(a, b) y(b, c)<z^{\prime}(c a)$ and $x(a, b) y^{\prime}(b, c)<z^{\prime}(c a)$, may be deduced at once from the results that have now been established. A few examples will be enough to illustrate the method.

$$
\left\{\epsilon(b a) \gamma(b c)<\beta^{\prime}(c a)\right\}^{\prime}<\left\{\beta(c a) \epsilon(b a)<\gamma^{\prime}(b c)\right\}^{\prime},
$$

by $\left(x y<z^{\prime}\right)^{\prime} \angle\left(z x<y^{\prime}\right)^{\prime}$;

$$
\begin{aligned}
\{\beta(a b) \epsilon(c b) & \left.<\gamma^{\prime}(c a)\right\}^{\prime}\left\{\beta(a b) \angle \gamma^{\prime}(a b)\right\} \\
& <\left\{\gamma^{\prime}(a b) \epsilon(c b)<\gamma^{\prime}(c a)\right\}^{\prime},
\end{aligned}
$$

by $(x y<z)^{\prime}(x<w)<(w y<z)^{\prime}$;

$$
\left\{\gamma^{\prime}(a b) \epsilon(c b) \angle \gamma^{\prime}(c a)\right\}^{\prime} \angle\{\epsilon(c b) \gamma(c a) \angle \gamma(a b)\}^{\prime},
$$

by $\left(x^{\prime} y<z^{\prime}\right)^{\prime}<(y z<x)^{\prime}$;

$$
\left\{\gamma^{\prime}(a b) \epsilon(c b)<\gamma^{\prime}(c a)\right\}^{\prime}<\left\{\gamma^{\prime}(a b) \gamma(c a)<\epsilon^{\prime}(c b)\right\}^{\prime},
$$

by $\left(x y<z^{\prime}\right)^{\prime} \angle\left(x z<y^{\prime}\right)^{\prime}$;

$$
\begin{gathered}
\left\{\epsilon(b a) \gamma(b c)<\beta^{\prime}(c a)\right\}^{\prime}\left\{\epsilon(c a) \angle \beta^{\prime}(c a)\right\} \\
\angle\{\epsilon(b a) \gamma(b c) \angle \epsilon(c a)\}^{\prime},
\end{gathered}
$$

by $(x y<z)^{\prime}(w<z)<(x y<w)^{\prime}$;

$\{\epsilon(b a) \gamma(b c) \angle \epsilon(c a)\}^{\prime}\{\epsilon(a c) \angle \epsilon(c a)\}<\{\gamma(b c) \epsilon(b a) \angle \epsilon(a c)\}^{\prime}$, by $(x y<z)^{\prime}(w<z)<(y x<w)^{\prime}$.

\section{EXERCISES}

1. Show that there exist no valid implications of the form $x^{\prime}(a, b)$ $y(b, c)<z(c a)$ or $x(a, b) y^{\prime}(b, c)<z(c a)$, and consequently none of the form, $x^{\prime}(a, b) y^{\prime}(b, c) \angle z(c a)$ or $x^{\prime}(a, b) y^{\prime}(b, c)<z^{\prime}(c a)$.

2 . Show that as a result of a complete induction of the moods in question (a) a valid mood of the syllogism, whose premises and conclusion are all unprimed forms and one of whose premises is of the same form as the conclusion, will remain valid, when the other premise is put in the $a$-form; and (b) a valid mood of the syllogism, whose premises are unprimed forms and whose conclusion is a primed form and one of whose premises is of a different form from the conclusion, will remain valid, when the other premise is put in the $a$-form. 


\section{CHAPTER VII}

$\S 36$. Because they possess similar properties, it is the custom to represent inclusion and implication-just as the negation of a class and the denial of a proposition-by the same sign, i. e.,

$$
\begin{aligned}
a \angle b & =a \text { is included in } b, \\
a \angle b^{\prime} & =a \text { is included in non-b, } \\
(a \angle b)^{\prime} & =a \text { is not included in } b, \text { etc. }
\end{aligned}
$$

"Let us take a pair of contrary names, as man and not-man. It is plain that between them they represent everything imaginable or real, in the universe. But the contraries of common language usually embrace, not the whole universe, but some one general idea. Thus, of men, Briton and alien are contraries: every man must be one of the two, no man can be both. Not-Briton and alien are identical names, and so are not-alien and Briton. The same may be said of integer and fraction among numbers, peer and commoner among subjects of the realm, male and female among animals, and so on, In order to express this, let us say that the whole idea under consideration is the universe (meaning merely the whole of which we are considering parts) and let names which have nothing in common, but which between them contain the whole idea under consideration, be called contraries in, or with respect to, that universe. Thus, the universe being mankind, Briton and alien are contraries, as are soldier and civilian, male and female, etc.: the universe being animal, man and brute are contraries, etc." (De Morgan, Formal Logic, pp. 37-38.)

Suppose that we should wish to express the propositional functions, $a, \beta, \gamma$ and $\epsilon$, in the forms that are ordinarily employed. It would then seem natural to recognize the following identities:

$$
\begin{aligned}
& a(a b)=(a \angle b)(b \angle a), \\
& \beta(a b)=(a \angle b)^{\prime}(b \angle a)^{\prime}\left(a \angle b^{\prime}\right)^{\prime}, \\
& \gamma(a b)=(a \angle b)(b \angle a)^{\prime}, \\
& \epsilon(a b)=\left(a \angle b^{\prime}\right) .
\end{aligned}
$$

We might then inquire if these representations verify all of the implications set down as true in the preceding chapters. In order to make this verification complete, it would be enough to deduce the characteristic postulates of our system by means of the transformations of the class calculus, which we should, accordingly, assume as necessity requires. Thus we should have:
(1) $(c \angle b)(b \angle a) \angle(c \angle a)$.
(2) $(a \angle b)(b \angle c) \angle(a \angle c)$. 
Multiplying together both sides of (1) and (2) and rearranging the factors conjoined in the antecedent,

$$
(b \angle a)(a \angle b)(c \angle b)(b \angle c) \angle(c \angle a)(a \angle c) \text {, }
$$

which by definition would be the same as

$$
a(b a) a(c b)<a(c a) \text {. }
$$

But it would soon be discovered that some of our implications break down. Thus,

would be rendered by

$$
\gamma(a b) \epsilon(a b)<o,
$$

$$
\begin{aligned}
& (a \angle b)(b \angle a)^{\prime}\left(a \angle b^{\prime}\right) \angle 0, \\
& \text { or }(a \angle b)\left(a \angle b^{\prime}\right) \angle(b \angle a),
\end{aligned}
$$

and this implication is manifestly not true in general.

§37. With the development of the class calculus a breakdown similar to the one just noticed was pointed out among the implications of the classical logic and it has been the habit of logicians to assert that the relation of subalternation and some of the valid moods of the syllogisms are fallacious. This misapprehension is all but universally shared by recent writers. Its removal may be effected by a solution similar to the one which follows. (See also Chap. IX.)

§38. Our rendering of the four propositional functions, $\alpha, \beta, \gamma$ and $\epsilon$, has been over-simplified. Let us attach to them, not the meaning which they had above and which has proven insufficient, but the one which follows, viz.,

$$
\begin{aligned}
& a(a b)=(a \angle b)(b \angle a), \\
& \beta(a b)=(a \angle b)^{\prime}(b \angle a)^{\prime}\left(a \angle b^{\prime}\right)^{\prime}, \\
& \gamma(a b)=(a \angle b)(b \angle a)^{\prime}\left(A^{\prime}+B\right), \\
& \epsilon(a b)=\left(a \angle b^{\prime}\right) A^{\prime} B^{\prime},
\end{aligned}
$$

where ${ }^{1} A=a \angle a^{\prime}$ and $B=b \angle b^{\prime}$.

Consider the syllogism $(\gamma \epsilon \epsilon)_{2}$. We have

$$
\begin{aligned}
& \text { (1) }(b \angle a)^{\prime}\left(b \angle b^{\prime}\right)^{\prime} A^{\prime} C^{\prime} \angle A^{\prime} C^{\prime} \text {. } \\
& \text { (2) }(a \angle b)\left(b \angle c^{\prime}\right) \angle\left(a \angle c^{\prime}\right) \text {. }
\end{aligned}
$$

Multiplying together both sides of (1) and (2) and factoring and

1 The assertion of $a \angle a^{\prime}$ is the same as to assert that $a$ is a null-class, or a class that contains no objects. The class contradictory to the null-class is called the universe. These two may be represented by the symbols 0 and $i$ respectively and are defined by $0 \angle i,(i<o)^{\prime}$. 
strengthening the antecedent, remembering that $\left(A^{\prime}+B\right) B^{\prime} C^{\prime} \angle$ $A^{\prime} C^{\prime}$,

$(a \angle b)(b \angle a)^{\prime}\left(A^{\prime}+B\right)\left(b \angle c^{\prime}\right) B^{\prime} C^{\prime} \angle\left(a \angle c^{\prime}\right) A^{\prime} C^{\prime}$ or $\gamma(a b) \epsilon(c b)<\epsilon(c a)$, since $\left(b \angle c^{\prime}\right)=\left(c \angle b^{\prime}\right)$ and $\left(a \angle c^{\prime}\right)=\left(c \angle a^{\prime}\right)$.

It will be easy to see that the characteristic features of the system, which we have set down, are now retained. Thus, $a(a b), \beta(a b)$ and $\epsilon(a b)$ alone are simply convertible, $a(a b)$ becomes true and the other forms false when the terms are identified, and $\mathrm{k}(a b) \mathrm{w}(a b)=0$. Two illustrations will furnish the student the clue to a verification of the remaining postulates.

(a) $\left(B^{\prime}+A\right)\left(C^{\prime}+B\right)=B^{\prime} C^{\prime}+A C^{\prime}+A B$, $B^{\prime} C^{\prime}+A C^{\prime}<C^{\prime}$, $A B<A$,

$\therefore$ (1) $\left(B^{\prime}+A\right)\left(C^{\prime}+B\right) \angle\left(C^{\prime}+A\right)$.

$(c \angle b)(b \angle a)<(c \angle a)$, $(a \angle b)^{\prime}(c \angle b)<(a \angle c)^{\prime}$,

$\therefore$ (2) $(b \angle a)(a \angle b)^{\prime}(c \angle b)(b \angle c)^{\prime} \angle(c \angle a)(a \angle c)^{\prime}$.

Multiplying together both sides of (1) and (2),

$(b \angle a)(a \angle b)^{\prime}\left(B^{\prime}+A\right)(c \angle b)(b \angle c)^{\prime}\left(C^{\prime}+B\right) \angle(c \angle a)$ $(a \angle c)^{\prime}\left(C^{\prime}+A\right)$, or $\gamma(b a) \gamma(c b)<\gamma(c a)$.

(b) $(b \angle a)\left(b \angle a^{\prime}\right)<B$, $\therefore(b \angle a)(b \angle c)\left(c \angle a^{\prime}\right)<B$, $\therefore(b \angle a)(b \angle c) B^{\prime} \angle\left(c \angle a^{\prime}\right)^{\prime}$,

$\therefore$ (1) $\quad(b \angle a)(a \angle b)^{\prime}(b \angle c)(c \angle b)^{\prime} B^{\prime} \angle\left(c \angle a^{\prime}\right)^{\prime}$ $\left(A+B^{\prime}\right)\left(C+B^{\prime}\right)=A C+B^{\prime} C+A B^{\prime}+B^{\prime}$, $A C+B^{\prime} C<C$, $A B^{\prime}<A$, $\therefore A C+B^{\prime} C+A B^{\prime} \angle A+C$.

$\therefore$ (2) $(b \angle a)(a \angle b)^{\prime}(b \angle c)(c \angle b)^{\prime}\left(A C+B^{\prime} C+A B^{\prime}\right) \angle$ $(A+C)$.

Adding together both sides of (1) and (2),

$(b \angle a)(a \angle b)^{\prime}\left(A+B^{\prime}\right)(b \angle c)(c \angle b)^{\prime}\left(C+B^{\prime}\right) \angle\left(c \angle a^{\prime}\right)^{\prime}$ $+A+C$ or $\gamma(b a) \gamma(b c)<\epsilon^{\prime}(c a)$.

\$39. The postulates (p. 43) may be established empirically by translating them into the new mode of representation and assigning appropriate concrete meanings to the terms. Thus, 


$$
\begin{array}{r}
\gamma(b a) \gamma(c b)<\gamma^{\prime}(c a), \\
\text { or } \gamma(b a) \gamma(c b) \gamma(c a)<0, \\
\text { implies } \gamma(b a) \gamma(c b)<0,
\end{array}
$$

since $\gamma(c a)$ may be strengthened at once to $\gamma(b a) y(c b)$, and the empirical untruth of this implication is manifest.

Translating $\gamma(b a) \gamma(c b) \gamma(c a)<0$ into the new mode of representation, we should have:

$$
\begin{gathered}
(b \angle a)(a \angle b)^{\prime}(c \angle b)(b \angle c)^{\prime}(c \angle a)(a \angle c)^{\prime} \\
\left(A+B^{\prime}\right)\left(B+C^{\prime}\right)\left(A+C^{\prime}\right) \angle o .
\end{gathered}
$$

Now, $(c \angle b)(b \angle a)<(c \angle a)$;

$\therefore$ we may omit the factor $(c<a)$.

And $(b \angle a)(b \angle c)^{\prime} \angle(a \angle c)^{\prime}$;

$\therefore$ we may omit the factor $(a<c)^{\prime}$.

And $\left(A+B^{\prime}\right)\left(B+C^{\prime}\right) \angle\left(A+C^{\prime}\right)$;

$\therefore$ we may omit the factor $\left(A+C^{\prime}\right)$.

The result $(b \angle a)(a \angle b)^{\prime}(c \angle b)(b \angle c)^{\prime}\left(A+B^{\prime}\right)\left(B+C^{\prime}\right) \angle$ $o$, yields,

$$
\begin{aligned}
& (b \angle a)(a \angle b)^{\prime}(c \angle b)(b \angle c)^{\prime} A B \angle 0, \\
& (b \angle a)(a \angle b)^{\prime}(c \angle b)(b \angle c)^{\prime} A C^{\prime} \angle 0, \\
& (b \angle a)(a \angle b)^{\prime}(c \angle b)(b \angle c)^{\prime} B^{\prime} C^{\prime} \angle 0 .
\end{aligned}
$$

Suppose that $a, b$ and $c$ be particularized thus:

Let $a=$ figures of less than six sides,

$b=$ figures of less than five sides,

$c=$ figures of less than four sides.

Each proposition conjoined in the antecedent of the last of the three implications above, for extra-logical reasons, becomes true and the untruth of the implication as a whole is manifest.

$\S 40$. It must be observed, however, that the interpretation of our four propositional functions, which has just been given, is not unique. All the implications of the logic we have been developing will hold, if the following meaning were to be assigned to the four forms, viz.,

$$
\begin{aligned}
& a(a b)=(a \angle b)(b \angle a)\left(A B+A^{\prime} B^{\prime}\right), \\
& \beta(a b)=(a \angle b)^{\prime}(b \angle a)^{\prime}\left\{\left(a \angle b^{\prime}\right)^{\prime}+(A+B)\right\}, \\
& \gamma(a b)=(a \angle b)\left\{(b \angle a)^{\prime}\left(A^{\prime}+B\right)+A^{\prime} B\right\}, \\
& \epsilon(a b)=\left(a \angle b^{\prime}\right) A^{\prime} B^{\prime} .
\end{aligned}
$$

Let one further illustration suffice:

$$
\begin{aligned}
& A B C+B^{\prime} C^{\prime} \angle A+C^{\prime}, \\
& (b \angle c)(c \angle b)(a \angle b)^{\prime} \angle(a \angle c)^{\prime},
\end{aligned}
$$


$\therefore(1)(b \angle c)(c \angle b)\left\{\left(A B C+B^{\prime} C^{\prime}\right)(a \angle b)^{\prime}\right\} \angle(a \angle c)^{\prime}$ $\left(A+C^{\prime}\right)$. $A B^{\prime} C^{\prime}<A C^{\prime}$

$\therefore$ (2) $(b \angle c)(c \angle b) A B^{\prime} C^{\prime} \angle A C^{\prime}$.

Adding together (1) and (2),

$(b \angle c)(c \angle b)\left\{\left(A B C+B^{\prime} C^{\prime}\right)(a \angle b)^{\prime}+A B^{\prime} C^{\prime}\right\}<(a \angle c)^{\prime}$ $\left(A+C^{\prime}\right)+A C^{\prime}$

and $(c \angle b)(b \angle a)<(c \angle a)$

$\therefore(b \angle a)\left\{(a \angle b)^{\prime}\left(A+B^{\prime}\right)+A B^{\prime}\right\}(c \angle b)(b \angle c)\left(B C+B^{\prime} C^{\prime}\right)$

$\angle(c \angle a)\left\{(a \angle c)^{\prime}\left(A+C^{\prime}\right)+A C^{\prime}\right\}$,

or $\gamma(b a) a(c b)<\gamma(c a)$. 


\section{CHAPTER VIII}

$\S 41$. In the last chapter it was remarked that some of the implications of the logic that we have been developing would break down, if we were to accept a traditional interpretation of our propositional functions. In that case we should come upon a logic whose characteristic postulates would stand in contradiction to those which we have become accustomed to accept.

It is important to observe that this circumstance does not compel us to conclude that one of the two systems must therefore be false in the absolute sense of that word. If one system is taken to be true, the other is by implication false, but it may well be that the world of common experience could force neither one of them upon us. In point of fact more than one system of inference is possible. A system of logic whose characteristic postulates stand in contradiction to those of the classical logic but which finds its application in precisely the same world, may be called appropriately a non-Aristotelian logic.

\$42. As an illustration of method we shall indicate in a set of exercises the existence of a system, whose underlying assumptions appear paradoxical to ordinary intuition, for this system will deny the truth of the proposition, all $a$ is all $a$, and assert that the proposition, no $a$ is $a$, is not untrue for all meanings of $a$.

In the exercises below the following meaning is to be attached to the categorical forms:

$$
\begin{aligned}
& a(a b)=(a \angle b)(b \angle a) A^{\prime} B^{\prime} \\
& \beta(a b)=(a \angle b)^{\prime}(b \angle a)^{\prime}\left(a \angle b^{\prime}\right)^{\prime} \\
& \gamma(a b)=(a \angle b)(b \angle a)^{\prime}\left(A^{\prime}+B\right) \\
& \epsilon(a b)=\left(a \angle b^{\prime}\right)+A+B
\end{aligned}
$$

EXERCISES

(1) If $\mathrm{k}(a b)$ and $\mathrm{w}(a b)$ can represent any one of the unprimed forms but can not represent the same form, $\gamma(a b)$ and $\gamma(b a)$ being considered distinct, show in what cases $\mathrm{k}(a b) \mathrm{w}(a b)<0$.

(2) Prove that the members of the set listed below are null-forms (see note p. 46).

$$
\begin{aligned}
& \beta\left(a a^{\prime}\right) \quad \epsilon(i i) \\
& \gamma(o a) \quad \alpha(o o) \\
& \gamma(i o) \quad \gamma(o i)
\end{aligned}
$$


(3) Prove that the members of the following set are one-forms:

$$
\begin{array}{ll}
\epsilon(0 o) & a(i i) \\
\epsilon(o i) & \epsilon\left(a a^{\prime}\right) \\
\epsilon(o a) & \epsilon(a 0)
\end{array}
$$

(4) Show that the truth or untruth of the following propositions is contingent on the meaning of $a$ :

$$
\begin{array}{ll}
a(i a) & \epsilon(a i) \\
a(a a) & \epsilon(i a) \\
\epsilon(a a) & a(a i)
\end{array}
$$

(5) Show in what cases the twenty-nine moods of the ordinary syllogism, that have been already recognized as valid, are valid in the present system. 


\section{CHAPTER IX}

$\S 43$. We shall now indicate the relation of the system of logic which has been partially developed, to the classical science perfected in the Organon of Aristotle. ${ }^{1}$

The four categorical forms employed by the traditional logic and denoted by the letters, A, E, I, O, are:

$$
\begin{aligned}
\mathrm{A}(a b) & =\text { All } a \text { is } b, \\
\mathrm{E}(a b) & =\text { No } a \text { is } b, \\
\mathrm{I}(a b) & =\text { Some } a \text { is } b, \\
\mathrm{O}(a b) & =\text { Some } a \text { is not } b,
\end{aligned}
$$

the word some expressed before the subject of $I$ and $O$ and understood before the predicate of $\mathrm{A}$ and $\mathrm{I}$, being interpreted to mean some at least, possibly all.

The valid moods of the Aristotelian syllogism are conveniently remembered by means of the following mnemonic lines, the vowels in each separate word standing for the mood in question:

Barbara, Celarent, Darii, Ferioque prioris;

Cesare, Camestres, Festino, Baroko, secundae;

Tertia, Darapti, Disamis, Datisi, Felapton,

Bokardo, Ferison, habet; Quarta insuper addit

Bramantip, Camenes, Dimaris, Fesapo, Fresison.

This mnemonic first appears in the Summulae Logicales of Petrus Hispanus (afterwards Pope John XXI), who, however, does not profess to be the author of it. Several other versions are found in later writers. A Greek mnemonic of the same kind is inserted in editions of the Organon preceding that of Pacius. (From Mansel's Aldrich, Artis Logicae Rudimenta, cap. III, $\S 5$, note z.) The moods not listed are gotten by weakening the universal conclusions to particular conclusions (see example 5 at the end of this chapter).

\$44. The connection between the propositional functions, A, E, I, O, and the special forms, which have been employed in the text, may be conveniently assumed to be as follows:

$$
\begin{aligned}
\mathrm{A}(a b) & =a(a b)+\gamma(a b), \\
\mathrm{E}(a b) & =\epsilon(a b), \\
\mathrm{I}(a b) & =a(a b)+\beta(a b)+\gamma(a b)+\gamma(b a), \\
\mathrm{O}(a b) & =\epsilon(a b)+\beta(a b)+\gamma(b a) .
\end{aligned}
$$

1 For a concise outline of the traditional logic and good of its kind the student may consult Thomas Fowler, Elements of Deductive Logic, Oxford, 1905. An extended treatment of the problem of this chapter is to be found in the writer's Letters on Logic, Philadelphia, 1920. 
By actually performing the indicated multiplications and allowing the product $\mathrm{k}(a b) \mathrm{w}(a b)$ to drop out whenever it occurs we should be able to express $a, \beta, \gamma$ and $\epsilon$ in the members of the set, A, E, I, O, thus:

$$
\begin{aligned}
& a(a b)=\mathrm{A}(a b) \mathrm{A}(b a), \\
& \beta(a b)=\mathrm{I}(a b) \mathrm{O}(a b) \mathrm{O}(b a), \\
& \gamma(a b)=\mathrm{A}(a b) \mathrm{O}(b a), \\
& \epsilon(a b)=\mathrm{E}(a b) .
\end{aligned}
$$

\$ 45. In order that the classical system should hold true in all of its parts, certain characteristic conditions must be satisfied. Besides the moods Barbara and Celarent, we should have to have:

1. Corresponding to each member of the set, A, E, I, O, there is another member of the same set which stands for its contradictory;

2. The relation of subalternation, $\mathrm{A} \angle \mathrm{I}$, holds true;

3. The subject and predicate of $\mathrm{E}$ and $\mathrm{I}$ alone are simply convertible.

Today it is all but universally taken for granted that not all of these conditions hold for all meanings of the terms and it is usual to retain conditions (1) and (3) and to assert that condition (2) is not generally true.

"The untruth of the traditional moods of the syllogism, by means of which from two universal judgments one would deduce a particular judgment, has been recognized separately by Miss Ladd (1883), Schröder, Nagy, Peano, etc. It is one of the first and most remarkable results of the adoption of a logical ideography." (Padoa, La Logique Déductive, etc. Revue de Métaphysique et de Morale,-T. 20, ${ }^{\circ} 1$, 1912, p. 67, note.) See also the second citation in small type of $\$ 46$ below.

\$46. Employing the notation of Chapters VII and VIII, it would seem natural to recognize the following identities:

$$
\begin{aligned}
\mathrm{A}(a b) & =(a<b), \\
\mathrm{E}(a b) & =\left(a<b^{\prime}\right), \\
\mathrm{I}(a b) & =\left(a<b^{\prime}\right)^{\prime}, \\
\mathrm{O}(a b) & =(a<b)^{\prime} .
\end{aligned}
$$

Compare the citation given below, wherein we have replaced a part of the author's notation by our own.

"The universal affirmative: 'All $a$ is $b$ ' is rendered by the formulas

$$
(a \angle b)=(a=a b)=\left(a b^{\prime}=0\right)=\left(a^{\prime}+b=i\right)
$$

and the universal negative: 'No $a$ is $b$ ' by the formulas

$$
\left(a \angle b^{\prime}\right)=\left(a=a b^{\prime}\right)=(a b=0)=\left(a^{\prime}+b^{\prime}=i\right)
$$


The particular affirmative: 'Some $a$ is $b$ ', being the negation of the universal negative, is rendered by the formulas

$$
\left(a \angle b^{\prime}\right)^{\prime}=\left(a=a b^{\prime}\right)^{\prime}=(a b=0)^{\prime}=\left(a^{\prime}+b^{\prime}=i\right)^{\prime}
$$

and the particular negative: 'Some $a$ is not $b$ ', being the negation of the universal affirmative is rendered by the formulas

$$
(a \angle b)^{\prime}=(a=a b)^{\prime}=\left(a b^{\prime}=0\right)^{\prime}=\left(a^{\prime}+b=i\right)^{\prime \prime \prime}
$$

(Couturat, L'Algebre de la Logique, Paris, 1905, notes, pp. 82, 83.)

However, granting this meaning of $\mathrm{A}, \mathrm{E}, \mathrm{I}$ and $\mathrm{O}$, subalternation and some of the valid moods of the syllogism fail. But this interpretation of Aristotle's four forms is in no way forced upon us. The meaning set down below accords equally well with the usage of language and with common sense. Let us assume:

$$
\begin{aligned}
& \mathrm{A}(a b)=(a<b) A^{\prime} B^{\prime}, \\
& \mathrm{E}(a b)=\left(a<b^{\prime}\right)+A+B, \\
& \mathrm{I}(a b)=\left(a<b^{\prime}\right)^{\prime} A^{\prime} B^{\prime}, \\
& \mathrm{O}(a b)=(a<b)^{\prime}+A+B .
\end{aligned}
$$

The characteristic features of the Aristotelian system may now be verified. As an illustration of the method of verifying the twenty-four valid moods of the syllogism, let us select the mood Darapti:

"But with our definitions, All S is $\mathrm{P}$ does not imply Some $\mathrm{S}$ is" $\mathrm{P}$, since the first allows the non-existence of $S$ and the second does not; thus conversion per accidens becomes invalid, and some of the moods of the syllogism are fallacious, e. g. Darapti: All M is S, All M is P, therefore Some S is P, which fails if there is no M." (Bertrand Russell, Introduction to Mathematical Philosophy, London, 1919, p. 164.)

We have:

$$
\text { Thus, } \begin{aligned}
\mathrm{A}(b a) & =(b \angle a) B^{\prime} A^{\prime} \\
\mathrm{A}(b c) & =(b \angle c) B^{\prime} C^{\prime} \\
\mathrm{I}(c a) & =\left(c \angle a^{\prime}\right)^{\prime} C^{\prime} A^{\prime}
\end{aligned}
$$

$$
\begin{aligned}
& (b \angle a)\left(a \angle c^{\prime}\right) \angle\left(b \angle c^{\prime}\right) \\
& (b \angle c)\left(c \angle b^{\prime}\right) \angle\left(b \angle b^{\prime}\right)
\end{aligned}
$$

Accordingly,

and

$$
\begin{aligned}
& (b \angle a)(b \angle c)\left(a \angle c^{\prime}\right) \angle\left(b \angle b^{\prime}\right) \\
& \text { since }\left(b \angle c^{\prime}\right)=\left(c \angle b^{\prime}\right)
\end{aligned}
$$

$$
\begin{aligned}
& (b \angle a)(b \angle c) B^{\prime} \angle\left(c \angle a^{\prime}\right)^{\prime} \\
& \text { since }\left(a \angle c^{\prime}\right)=\left(c \angle a^{\prime}\right)
\end{aligned}
$$


$\therefore(b \angle a)(b \angle c) A^{\prime} B^{\prime} C^{\prime} \angle\left(c \angle a^{\prime}\right)^{\prime} A^{\prime} C^{\prime}$

or $\left.\left.\left\{(b \angle a) B^{\prime} A^{\prime}\right\}\left\{(b \angle c) B^{\prime} C^{\prime}\right\} \angle\right\}\left(c \angle a^{\prime}\right)^{\prime} C^{\prime} A^{\prime}\right\}$

which by definition is the same as

$$
\mathrm{A}(b a) \mathrm{A}(b c)<\mathrm{I}(c a) \text {. }
$$

Exercises

(1) Verify the three conditions equivalent to those of $\$ 44$, viz.,

$$
\begin{aligned}
& \text { 1. } \mathrm{A}(a b) \mathrm{O}(a b)<0 \\
& \mathrm{E}(a b) \mathrm{I}(a b)<0 \\
& \mathrm{~A}^{\prime}(a b) \mathrm{O}^{\prime}(a b)<0 \\
& \mathbf{E}^{\prime}(a b) I^{\prime}(a b)<0 \\
& \text { 2. } \mathrm{A}(a b) \mathrm{E}(a b)<0 \\
& \text { 3. } \mathrm{E}(a b) \mathrm{I}(b a)<0
\end{aligned}
$$

We might easily, had we wished, have interpreted $A, E, I$ and $O$ in $\$ 45$ so as to satisfy the additional conditions $\mathrm{A}^{\prime}(a a) \angle 0, \mathrm{E}(a a) \angle 0$ (see Exercise 3).

(2) Verify the twenty-four valid moods of the Aristotelian syllogism (see the paragraph in small type of $\$ 43$ ).

(3) Show that the truth or untruth of the following propositions is contingent on the meaning of $a$ :

$$
\mathrm{A}(a a) \quad \mathrm{E}(a a) \quad \mathrm{A}(a i)
$$

(4) If affirmative forms are those, which become false when the terms are made contradictory, and negative forms are those which become true under the same circumstances, show that $\mathrm{A}(a b)$ and $\mathrm{I}(a b)$ are affirmative and that $\mathrm{E}(a b)$ and $\mathrm{O}(a b)$ are negative forms.

(5) If $\mathrm{X}(a b)<\mathrm{Y}(a b)$ and $\{\mathrm{Y}(a b)<\mathrm{X}(a b)\}^{\prime}$, then $\mathrm{X}(a b)$ is said to be universal and $\mathrm{Y}(a b)$ is said to be particular. Show that $\mathrm{A}(a b)$ and $\mathrm{E}(a b)$ are universal and that $\mathrm{I}(a b)$ and $\mathrm{O}(a b)$ are particular forms.

(6) If affirmative forms are those that become true when the terms have been identified and negative forms are those that become false under the same circumstances, show that $\mathrm{A}(a b)$ and $\mathrm{I}(a b)$ are affirmative and that $\mathrm{E}(a b)$ and $\mathrm{O}(a b)$ are negative forms upon the following interpretation of $\mathrm{A}, \mathrm{E}, \mathrm{I}$ and $\mathrm{O}$ :

$$
\begin{gathered}
\mathrm{A}(a b)=(a \angle b)\left(A^{\prime}+B\right), \\
\mathrm{E}(a b)=\left(a<b^{\prime}\right)\left(A^{\prime}+B_{1}\right), \\
\mathrm{I}(a b)=\left(a<b^{\prime}\right)^{\prime}+A B_{1}^{\prime}, \\
\mathrm{O}(a b)=(a<b)^{\prime}+A B^{\prime}, \\
\text { where } B_{1}=b^{\prime} \angle b .
\end{gathered}
$$

(7) Assuming the equalities of Exercise 6, show that the relation of obversion, $\mathrm{A}(a b)=\mathrm{E}\left(a b^{\prime}\right)$, holds true.

(8) Assuming the equalities of Exercise 6, show that conditions 1 and 2 of $\$ 44$ are satisfied.

(9) By means of the same equalities verify the two moods Barbara and Celarent.

(10) From the two moods of Exercise 9 deduce ten other valid moods of the Aristotelian syllogism by means of the principles of Chapter VI. 
(11) Interpreting $A, E, I$ and $O$ as in exercises (1)-(5), establish the invalidity of the following moods of the syllogism,
$\mathrm{AAO}_{1}, \quad \mathrm{EEI}_{1}$,
$\mathrm{AAO}_{4}$
$\mathrm{AEI}_{1}, \quad \mathrm{OAO}_{1}$,
$\mathrm{AEO}_{1}, \quad \mathrm{AAA}_{4}$,

either by allowing the terms to take on the limiting values $o$ and $i$ in appropriate ways, or "empirically" by assigning to them other concrete meanings as in $\$ 39$, Chapter VII.

(12) From the seven moods listed above derive the remaining two hundred and twenty-five invalid moods by means of the principles of $\$ 34$, Chapter VI. 






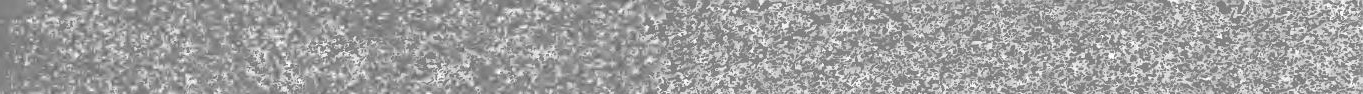

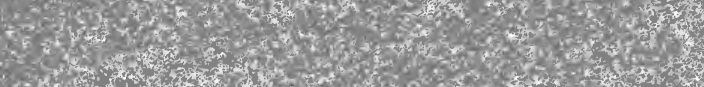

17.

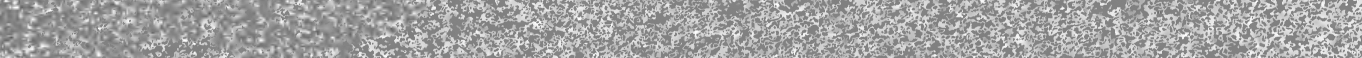
79.7. H.t.

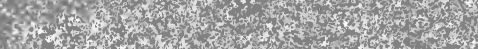
3.

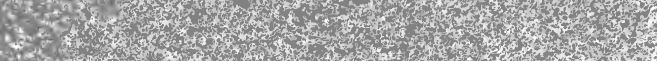

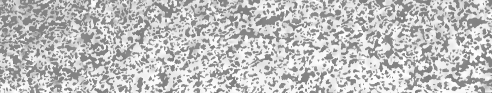
W

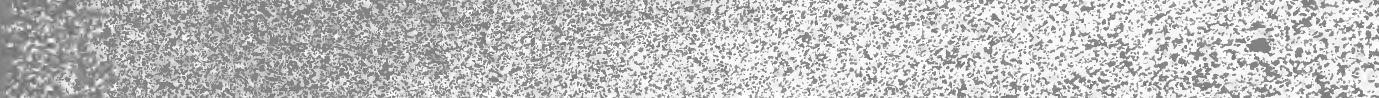

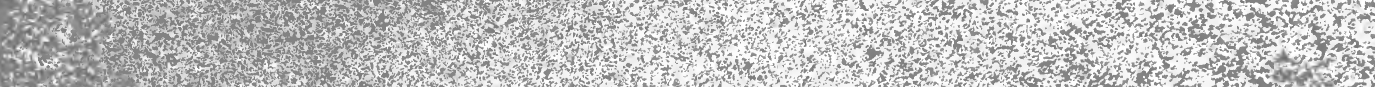

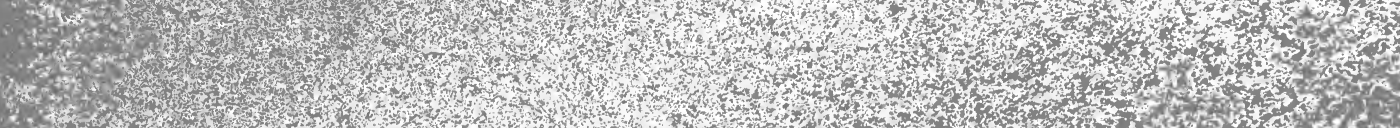

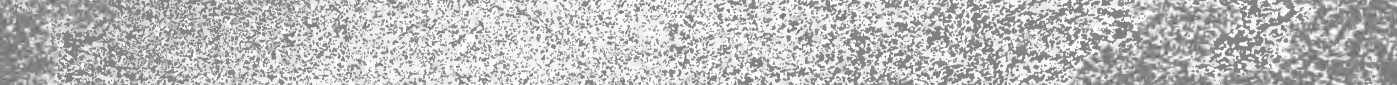

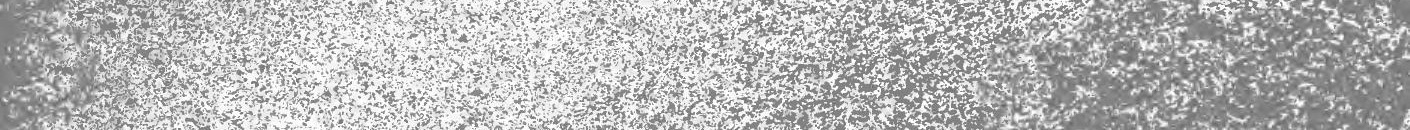

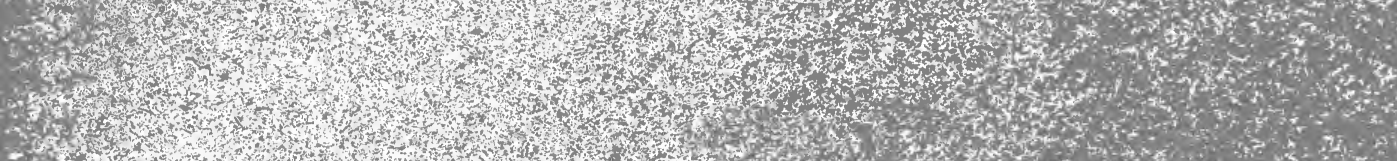

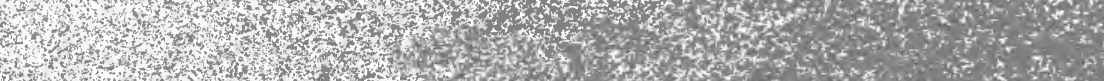
$\frac{3}{36}$

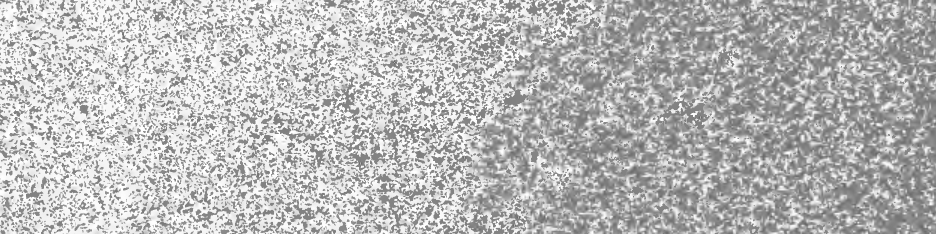

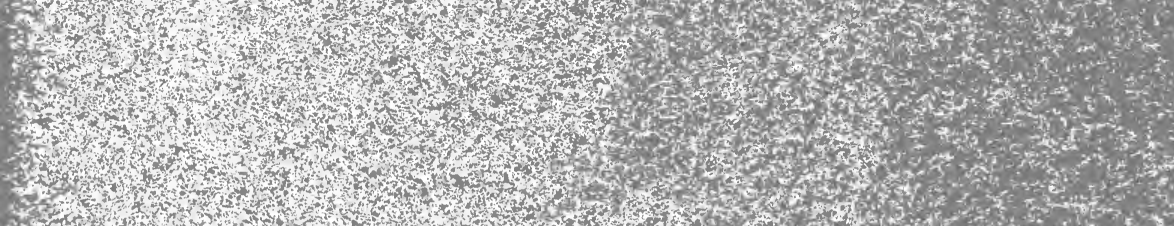

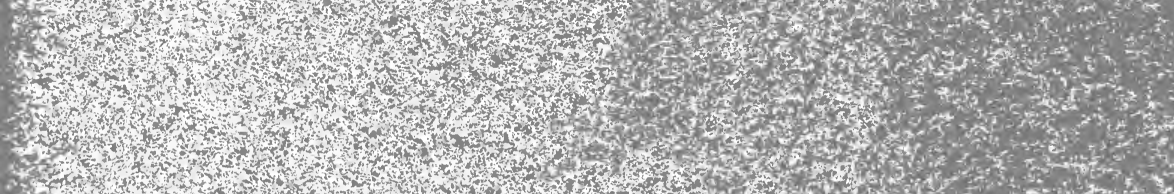
(1)

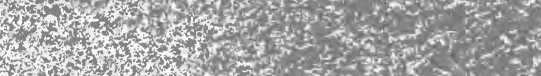

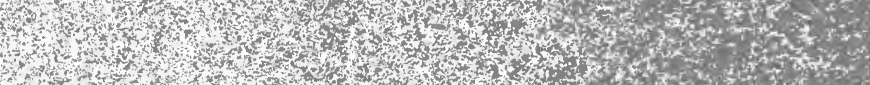

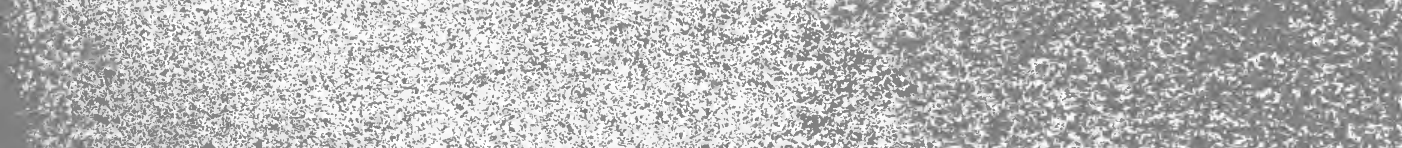

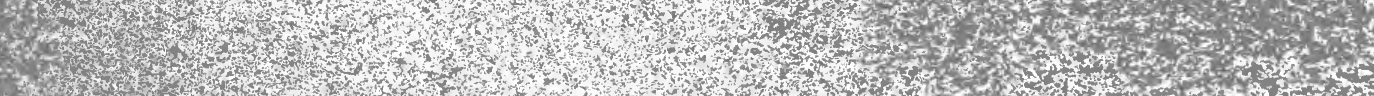

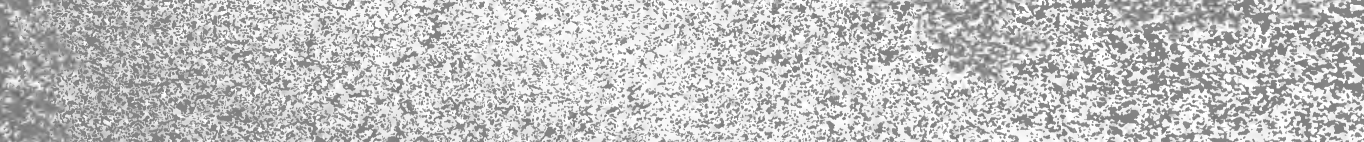
6.

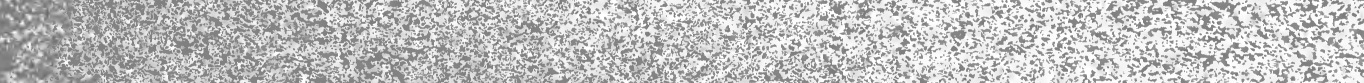

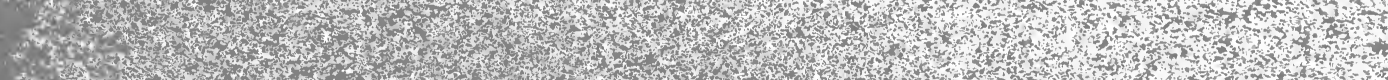

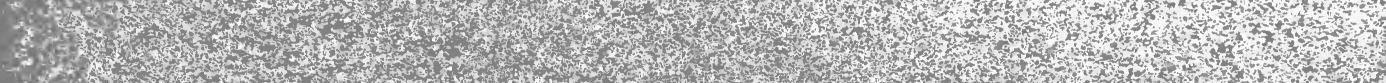

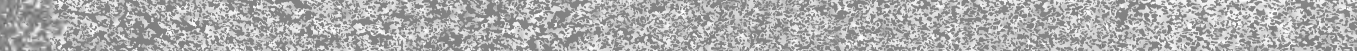

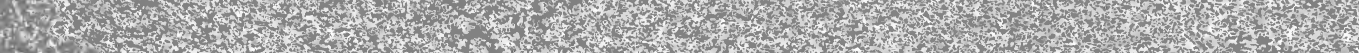


